$\xi=-$ 国

\title{
Transient Analysis of K-node Tandem Queuing Model with Load Dependent Service Rates
}

\author{
Sita Rama Murthy M.* ${ }^{1}$, Srinivasa Rao K. ${ }^{2}$, Ravindranath V. ${ }^{3}$, Srinivasa Rao P. ${ }^{4}$ \\ ${ }^{1}$ Dept.of Basic Science, Vishnu Institute of Technology, Bhimavaram -534202, Andhra Pradesh, INDIA. \\ ${ }^{2}$ Dept.of Statistics, Andhra University, Visakhapatnam-530003, Andhra Pradesh, INDIA. \\ ${ }^{3}$ Dept. of Mathematics, Jawaharlal Nehru Technological University Kakinada, Kakinada-533003, Andhra Pradesh, INDIA. \\ ${ }^{4}$ Dept. of Computer Science and Systems Engineering , Andhra University, Visakhapatnam-530003, Andhra Pradesh, INDIA. \\ *Corresponding author email : srmmushunuri@gmail.com,
}

\begin{abstract}
This paper deals with the development and analysis of K-node series and parallel queueing model with load dependent service rates. Here it is assumed that the customers arrive to the initial queue and waiting line for service. After completing the service at first service station they may join one of the (K-1) queues which are parallel and connected to first queue in series. After getting service from the service station they leave the system. Here it is assumed that the service rates in each service station are dependent on number of customers in the queue connected to it .The arrival and service completions in each queue are assumed to follow Poisson processes. Using difference-differential equations the joint probability function of number of customers in each queue are derived. The system performance measures such as average number of customers in each queue ,throughput of each service station ,the probability of idleness of each server ,the waiting time of customer in each queue are derived explicitly. The sensitivity of the model with respect to parameters are analysed through numerical illustration. It is observed that the state dependent service rates has significant influence on performance measures. This model also includes the earlier models as particular cases for specific values of the parameters. This model is useful in analysing the communication networks, transportation systems ,production processes and cargo handling.
\end{abstract}

Key words: Poisson process, Tandem queue, Load dependent, Forked queuing model, Performance of system.

\section{Introduction}

A Queue is a waiting line of units demanding service at a server facility. Erlang [1] pioneered the mathematical modelling of queueing system. There after several models have been developed and analysed in order to evaluate the performance of several systems for control and monitoring. Queueing models formulate a prerequisite for design and and development of several systems arising at places like Communication networks, ATM scheduling ,Transportation systems, Production processes etc.,(Boxima O.J., [2], Bunday.B.D., [3], SrinivasaRaoet.al., [4], [5], [6] , Charan Jeet Singh et.al., [7] ,Ramasundari et.al[8]).

Recently much work has been reported regarding Tandem Queueing models. In Tandem Queueing models the out put of one Queue formulate the input of the other.Jackson Paul[9], Srinivasa Rao et.al., [7] [5] [10],Che Soong Kim et.al[11], Raghavendran et.al[12] and others have developed various tandem Queueing models with the assumption that arrivals and services are independent. But in practical situations the service time is to adjusted depending upon the number of customers in the Queue. This type of Queueing models are called load dependent Queueing models. Srinivasa Rao et.al., [4], [5], Varma et.al., [13], Padmavathi et.al., [14], Nageswara Rao et.al., [15] Trinadha Rao et.al., [16], Suhasini et.al., [17], [18], [19], Rajasekhar Reddy et.al [20]have developed Queueing models with the assumption that the service rates are dependent on the number of customers in the Queue. In all these papers they assumed that the nodes are connected in tandem and single.

But in several practical situations after getting service from the first Queue the customer may join one of the several Queues connected to it for service .For example in Communication networks after getting service from the first transmitter the data/voice packets are to be routed to one of the several buffers connected in parallel for forward transmission. This type of scenario is also visible in Production processes such as Glass manufacturing, where the raw material is converted as liquid glass. It is then transferred to several production lines which are parallel for making different types of glass ware.This type of Queueing models may be called as 2-node series and K parallel Queueing systems, referred as forked Queueing models.

Little work has been reported regarding 2-node series and $\mathrm{K}$ parallel Queueing models which are useful for analysing several systems more close to the reality. Hence in this article we develop and analyse a forked Queueing model in which 2 nodes are in series and K Queues are in parallel. Here it is assumed that the arrival processes and service processes follow Poisson processes. It is further assumed that the service rate of each service station depends on the number of customers in the Queue connected to it. Using the difference -differential equations the joint probability generating function of the number of customers in each Queue is derived. The performance of the model is analysed by deriving explicit expressions for the system characteristics such as average number of customers in the Queue, Probability of idleness of each 
service station, Throughput of the nodes, Average waiting time customers in each Queue, Utilisation of each server etc., The sensitivity analysis of the model is carried with a numerical illustration

\section{Queueing Model with Load Dependent Service Rates}

In this section we consider queueing model with $\mathrm{K}$ buffers $B_{1}, B_{2}, \ldots B_{k}$ and $\mathrm{K}$ servers $S_{1}, S_{2}, \ldots S_{k}$ connected as forked network ,the capacity of buffers being infinite .we assume that the customers after getting service through first server may join any of the servers $S_{2}, S_{3}, \ldots S_{k}$ which are parallel and connected to first server in tandem i.e., the customers after getting served at $S_{1}$ may join second buffer with probability $\theta_{1}$ or third buffer with probability $\theta_{2}$ or $\mathrm{K}$ th buffer with probability $\theta_{k-1}$. Number of customers arriving at first buffer follows Poisson process with arrival rate $\lambda$ (parameter).Similarly number of customers served in servers follows Poisson process with parameters $\mu_{1}, \mu_{2}, \mu_{3} \ldots \mu_{k}$

Then difference differential equations governing the system are

$$
\begin{aligned}
& \frac{\partial P}{\partial t}\left(n_{1}, n_{2}, \ldots n_{k} ; t\right)=-\left[\lambda+\sum_{i=1}^{k} n_{i} \mu_{i}\right] P\left(n_{1}, n_{2}, ., n_{k} ; t\right) \\
& +\left(n_{1}+\right) \mu_{1}\left[\theta _ { 1 } P \left(n_{1}+1, n_{2}-1, n_{\left.3, \cdots, n_{k} ; t\right)+. .}\right.\right. \\
& \left.+\theta_{k-1} P\left(n_{1}+1, n_{2}, \ldots, n_{k}-1 ; t\right)\right] \\
& +\left(n_{2}+1\right) \mu_{2} P\left(n_{1}, n_{2}+1, n_{\left.3, . ., n_{k} ; t\right)+. .}\right. \\
& +\left(n_{k}+1\right) \mu_{k} P\left(n_{1}, n_{2} \ldots n_{k}+1 ; t\right)+\lambda P\left(n_{1}-1, n_{2, .}, n_{k}, t\right) \\
& \frac{\partial P}{\partial t}\left(0, n_{2}, \ldots n_{k} ; t\right)=-\left[\sum_{i=2}^{k} n_{i} \mu_{i}\right] P\left(0, n_{2,}, n_{k}, t\right) \\
& +\mu_{1}\left[\theta _ { 1 } P \left(1, n_{2}-1, n_{\left.3, \cdots, n_{k} ; t\right)+\ldots}\right.\right. \\
& \left.+\theta_{k-1} P\left(1, n_{2}, n_{3}, \ldots, n_{k}-1 ; t\right)\right] \\
& +\left(n_{2}+1\right) \mu_{2} P\left(0, n_{2}+1, n_{3, \cdots}, n_{k} ; t\right)+\text {.. } \\
& +\left(n_{k}+1\right) \mu_{k} P\left(0, n_{2}, n_{3}, \ldots, n_{k}+1 ; t\right) \\
& \frac{\partial P}{\partial t}\left(n_{1}, n_{2}, ., 0 ; t\right)=-\left[\lambda+\sum_{i=1}^{k-1} n_{i} \mu_{i}\right] P\left(n_{1}, n_{2, \cdot, 0, t}\right) \\
& +\left(n_{1}+1\right) \mu_{1}\left[\theta_{1} P\left(n_{1}+1, n_{2}-1, n_{3}, \ldots, 0 ; t\right)+\right. \\
& \left.\ldots+\theta_{k-2} P\left(n_{1}+1, n_{2}, \ldots, n_{k-1}-1,0 ; t\right)\right] \\
& +\left(n_{2}+1\right) \mu_{2} P\left(n_{1}, n_{2}+1, n_{3, \cdots, 0 ; t)}\right. \\
& +\left(n_{3}+1\right) \mu_{3} P\left(n_{1}, n_{2}, n_{3}+1, . .0 ; t\right)+\ldots+ \\
& \left(n_{k}+1\right) \mu_{k} P\left(n_{1}, n_{2} \ldots, 1 ; t\right)+\lambda P\left(n_{1}-1, n_{2, \cdot, 0, t)}\right. \\
& \frac{\partial P}{\partial t}\left(0,0, \ldots n_{k} ; t\right)=-\left[\lambda+\sum_{i=3,}^{k} n_{i} \mu_{i}\right] P\left(0,0 . ., n_{k}, t\right) \\
& +\mu_{1}\left[\theta_{2} P\left(1,0, n_{3}-1, \ldots, n_{k} ; t\right)+\cdots\right. \\
& \left.+\theta_{k-1} P\left(1,0, \ldots, n_{k}-1 ; t\right)\right] \\
& +\mu_{2} P\left(0,1, n_{3,}, n_{k} ; t\right)+\ldots \\
& +\left(n_{k}+1\right) \mu_{k} P\left(0,0, n_{3} \ldots, n_{k}+1 ; t\right) \\
& \frac{\partial \mathrm{P}}{\partial \mathrm{t}}\left(\mathrm{n}_{1}, \mathrm{n}_{2}, . ., 0,0 ; \mathrm{t}\right)=-\left[\lambda+\sum_{\mathrm{i}=1}^{\mathrm{k}-2} \mathrm{n}_{\mathrm{i}} \mu_{\mathrm{i}}\right] \mathrm{P}\left(\mathrm{n}_{1}, \mathrm{n}_{2,}, ., 0,0 ; \mathrm{t}\right)
\end{aligned}
$$

Let

$P\left(n_{1}, n_{2}, \cdots, n_{k} ; t\right)=$

$\sum_{n_{1}=0}^{\infty} \sum_{n_{2}=0}^{\infty} \ldots \sum_{n_{k}=0}^{\infty} p\left(n_{1}, n_{2}, \ldots n_{k} ; t\right) s_{1}{ }^{n_{1}} S_{2}{ }^{n_{2}} \ldots s_{k}{ }^{n_{k}}$ be probability generating function of $p\left(n_{1}, n_{2}, \ldots n_{k} ; t\right)$.Multiplying equations (1)..(8)with probability generating function and summing over $n_{1}, n_{2}, \ldots n_{k}$ from 0 to $\infty$ we get Joint Probability generating function of number of customers in first, second,.... $\mathrm{k}^{\text {th }}$ buffers respectively at any time $\mathrm{t}$ as

$$
\begin{aligned}
& P\left(n_{1}, n_{2}, \ldots n_{k} ; t\right)=\exp \left[\lambda \left\{\frac{1}{\mu_{1}}\left(n_{1}-1\right)\left(1-e^{-\mu_{1} t}\right)+\right.\right. \\
& \frac{\theta_{1}}{\mu_{2}}\left(n_{2}-1\right)\left(1-e^{-\mu_{2} t}\right)+. .+\frac{\theta_{k}}{\mu_{k}}\left(n_{k}-1\right)\left(1-e^{-\mu_{k} t}\right)+ \\
& \left.\left.\frac{\theta_{1}}{\left(\mu_{2-} \mu_{1}\right)}\left(n_{2}-1\right)+. .+\frac{\theta_{k-1}}{\left(\mu_{k-} \mu_{1}\right)}\left(n_{k}-1\right)\left(e^{-\mu_{k} t}-e^{-\mu_{1} t}\right)\right\}\right]
\end{aligned}
$$

respectively. It is also assumed that service rate in each server is linearly dependent on the content of buffer connected to it. The queue discipline is first come first serve (FCFS)

The schematic diagram representing the queuing model is shown in fig. 1

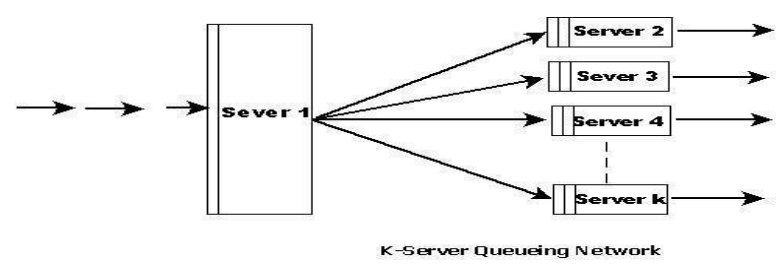

Figure 1: K-Server Queueing Network

Let $P\left(n_{1}, n_{2}, ., n_{k} ; t\right)$ be the probability that there are $\mathrm{n}_{1}$ costumers in first buffer, $\mathrm{n}_{2}$ customers in second buffer and $\mathrm{n}_{\mathrm{k}}$ customers in $\mathrm{k}$ th buffer.

$$
\begin{gathered}
+\left(\mathrm{n}_{1}+1\right) \mu_{1}\left[\theta_{1} \mathrm{P}\left(\mathrm{n}_{1}+1, \mathrm{n}_{2}-1, \ldots, \mathrm{n}_{\mathrm{k}-2}, 0,0 ; \mathrm{t}\right)+\right. \\
\left.. .+\theta_{\mathrm{k}-3} \mathrm{P}\left(\mathrm{n}_{1}+1, \mathrm{n}_{2}, ., \mathrm{n}_{\mathrm{k}-2}-1,0,0 ; \mathrm{t}\right)\right] \\
\left(\mathrm{n}_{2}+1\right) \mu_{2} \mathrm{P}\left(\mathrm{n}_{1}, \mathrm{n}_{2}+1, \mathrm{n}_{3, .}, \mathrm{n}_{\mathrm{k}-2}, 0,0 ; \mathrm{t}\right)+. . \\
+\left(\mathrm{n}_{\mathrm{k}-2}+1\right) \mu_{\mathrm{k}} \mathrm{P}\left(\mathrm{n}_{1}, \mathrm{n}_{2} . ., \mathrm{n}_{\mathrm{k}-2}+1,0,0 ; \mathrm{t}\right) \\
+\lambda \mathrm{P}\left(\mathrm{n}_{1}-1, \mathrm{n}_{2, .}, \mathrm{n}_{\mathrm{k}-2}, 0,0 ; \mathrm{t}\right)
\end{gathered}
$$

$$
\begin{aligned}
& \frac{\partial \mathrm{P}}{\partial \mathrm{t}}\left(0,0,0, \mathrm{n}_{4}, \ldots \mathrm{n}_{\mathrm{k}} ; \mathrm{t}\right)=-\left[\lambda+\sum_{\mathrm{i}=4}^{\mathrm{k}} \mathrm{n}_{\mathrm{i}} \mu_{\mathrm{i}}\right] \mathrm{P}\left(0,0,0, \mathrm{n}_{4, \cdots}, \mathrm{n}_{\mathrm{k}}, \mathrm{t}\right) \\
& +\mu_{1}\left[\theta_{3} \mathrm{P}\left(1,0,0, \mathrm{n}_{4}-1, \mathrm{n}_{3,}, \ldots, \mathrm{n}_{\mathrm{k}} ; \mathrm{t}\right)+\ldots\right. \\
& \left.+\theta_{\mathrm{k}-1} \mathrm{P}\left(1,0,0, \mathrm{n}_{4}, \ldots, \mathrm{n}_{\mathrm{k}}-1 ; \mathrm{t}\right)\right] \\
& +\mu_{2} \mathrm{P}\left(0,1,0, \mathrm{n}_{4, .}, \mathrm{n}_{\mathrm{k}} ; \mathrm{t}\right)+. . \\
& +\left(\mathrm{n}_{\mathrm{k}}+1\right) \mu_{\mathrm{k}} \mathrm{P}\left(0,0,0, \mathrm{n}_{4}, \ldots, \mathrm{n}_{\mathrm{k}}+1 ; \mathrm{t}\right)
\end{aligned}
$$

$\frac{\partial \mathrm{P}}{\partial \mathrm{t}}\left(\mathrm{n}_{1}, . ., \mathrm{n}_{\mathrm{k}-3}, 0,0,0 ; \mathrm{t}\right)=-[\lambda+$

$\left.\sum_{\mathrm{i}=1}^{\mathrm{k}-3} \mathrm{n}_{\mathrm{i}} \mu_{\mathrm{i}}\right] \mathrm{P}\left(\mathrm{n}_{1} \ldots, \mathrm{n}_{\mathrm{k}-3}, 0,0,0: \mathrm{t}\right)+\left(\mathrm{n}_{1}+\right.$

1) $\mu_{1}\left[\theta_{1} P\left(n_{1}+1, n_{2}-1, \ldots, n_{k-3}, 0,0,0 ; t\right)+\right.$

$\left.\ldots+\theta_{\mathrm{k}-4} \mathrm{P}\left(\mathrm{n}_{1}+1, \mathrm{n}_{2}, ., \mathrm{n}_{\mathrm{k}-3}-1,0,0,0 ; \mathrm{t}\right)\right]+$

$\left(\mathrm{n}_{2}+1\right) \mu_{2} \mathrm{P}\left(\mathrm{n}_{1}, \mathrm{n}_{2}+1, \mathrm{n}_{3,}, \mathrm{n}_{\mathrm{k}-3}, 0,0,0 ; \mathrm{t}\right)+$

$\ldots+\mu_{\mathrm{k}} \mathrm{P}\left(\mathrm{n}_{1}, \mathrm{n}_{2} \ldots, \mathrm{n}_{\mathrm{k}-3}, 0,0, \mathrm{n}_{\mathrm{k}}+1 ; \mathrm{t}\right)$

$+\lambda \mathrm{P}\left(\mathrm{n}_{1}-1, \mathrm{n}_{2,}, \cdot \mathrm{n}_{\mathrm{k}-3}, 0,0,0 ; \mathrm{t}\right)$

$\frac{\partial P}{\partial t}(0,0, . .0 ; t)=-\lambda P(0,0, . .0 ; t)+\mu_{2} P(0,1,0, . .0 ; t)+$

$\mu_{3} \mathrm{P}(0,0,1,0, \ldots, 0 ; \mathrm{t})+\ldots+\mu_{\mathrm{k}} \mathrm{P}(0,0, . .0,1 ; \mathrm{t})$

\section{Characteristics of the Model}

$$
\begin{aligned}
& \text { Putting } n_{1}=0, n_{2,}=0, \ldots . n_{k}=0 \text { in (9) we get } \\
& \qquad \begin{array}{l}
\quad P(0,0,0 \ldots 0 ; t)= \\
\exp \left[-\lambda\left\{\frac{1}{\mu_{1}}\left(1-e^{-\mu_{1} t}\right)+\frac{\theta_{1}}{\mu_{2}}\left(1-e^{-\mu_{2} t}\right)+.\right.\right. \\
+\frac{\theta_{k}}{\mu_{k}}\left(1-e^{-\mu_{k} t}\right) \frac{\theta_{1}}{\left(\mu_{2-} \mu_{1}\right)}+\left(e^{-\mu_{2} t}-e^{-\mu_{1} t}\right)+. . \\
\left.\left.+\frac{\theta_{2}}{\left(\mu_{3-} \mu_{1}\right)}\left(e^{-\mu_{3} t}-e^{-\mu_{1} t}\right)+\frac{\theta_{k-1}}{\left(\mu_{k-} \mu_{1}\right)}\left(e^{-\mu_{k} t}-e^{-\mu_{1} t}\right)\right\}\right]
\end{array}
\end{aligned}
$$

Which gives the probability that the queue is empty at any time t. 


\subsection{Performance Analysis of First Buffer}

Putting $n_{2}=1, n_{3}=1, \ldots . n_{k}=1$ in (9) we get probability generating function of first buffer size distribution as $P\left(n_{1} ; t\right)=$ $\exp \left[\lambda\left\{\frac{1}{\mu_{1}}\left(n_{1}-1\right)\left(1-e^{-\mu_{1} t}\right)\right\}\right]$

Mean number of customers in first buffer is

$\mathrm{E}\left(N_{1}\right)=L_{1}(t)=\frac{\lambda}{\mu_{1}}\left(1-e^{-\mu_{1} t}\right)$

Variation in number of customers in first buffer is

$\operatorname{Var}\left(N_{1}\right)=\frac{\lambda}{\mu_{1}}\left(1-e^{-\mu_{1} t}\right)$

Putting $n_{1}=0$ in (11) we get the probability that the first buffer is empty as

Throughput of first server is Thp1 $(\mathrm{t})=\mu_{1} \cdot U_{1}$

$=\mu_{1}\left[1-\exp \left\{\frac{-\lambda}{\mu_{1}}\left(1-e^{-\mu_{1} t}\right)\right\}\right]$

Average waiting time of customers in first buffer ( delay in first server)is $W_{1}(t)=\frac{L_{1}(t)}{T h p_{1}(t)}=\frac{\frac{\lambda}{\mu_{1}}\left(1-e^{-\mu_{1} t}\right)}{\mu_{1}\left[1-\exp \left\{\frac{-\lambda}{\mu_{1}}\left(1-e^{-\mu_{1} t}\right)\right\}\right]}$

\subsection{Performance Analysis of $i^{\text {th }}$ Buffer for $i=2,3, . . k$}

Putting $n_{1}=1, n_{2}=1, n_{3}=1, \ldots n_{k-1}=1$ in (9) we get probability generating function of $\mathbf{i}^{\text {th }}$ buffer size distribution as

$$
\begin{aligned}
& P\left(n_{i} ; t\right)=\exp \left[\lambda \left\{\frac{\theta_{i-1}}{\mu_{i}}\left(n_{i}-1\right)\left(1-e^{-\mu_{i} t}\right)+\frac{\theta_{i-1}}{\left(\mu_{i-} \mu_{1}\right)}\left(n_{i}-\right.\right.\right. \\
& \text { 1) } \left.\left.\left(e^{-\mu_{i} t}-e^{-\mu_{1} t}\right)\right\}\right] \\
& P(0, . . . . ; t)=\exp \left[\frac{-\lambda}{\mu_{1}}\left(1-e^{-\mu_{1} t}\right)\right]
\end{aligned}
$$

Utilization of first server is $U_{1}=1-P(0, ., \ldots$, ; $t)$

\section{Numerical Illustration}

The transient behaviour of the model is studied by computing the performance measures with the following set of values for the system parameters.

$$
\begin{aligned}
t=0.1,0.2,0.3,0.4,0.5 ; \lambda=10,11,12,13,14 ; \mu_{i} \\
=10,11,12,13,14 ; i=1,2,3,4 ; \theta_{j} \\
=0.1,0.2,0.3,0.4,0.5 ; j=1,2
\end{aligned}
$$

Each of the parameters $t, \lambda, \mu_{1}, \mu_{2}, \mu_{3}, \mu_{4}, \theta_{1}, \theta_{2}, \theta_{3}$ are varied one at a time keeping all other fixed the mean number of customers in each buffer $L_{1}, L_{2}, L_{3}, L_{4}$ is calculated along with mean number of customers $L(t)$ in the entire system and the calculations are recorded in Table1.The corresponding probability for emptiness of each server and also the utilization of servers are calculated for each value of parameters and tabulated in Table2.The throughputs of four servers $T h p_{1}, T h p_{2}, T h p_{3}, T h p_{4}$ along with mean waiting times of customers in four buffers $W_{1}, W_{2}, W_{3}, W_{4}$ are calculated and tabulated in Table 3.

From Table1, we observe that as time $t$ increases from 0.1 to 0.5 there is increase in mean number of customers in each buffer. The same phenomenon can be observed with mean number of customers in the entire system. Thus if service rate $\mu_{1}$ is increased keeping $\mu_{2}, \mu_{3}, \mu_{4}$ unchanged the corresponding buffer at first server $L_{1}(t)$ gets decreased. Correspondingly the pressure on
Mean number of customers in $\mathbf{i}^{\text {th }}$ buffer is $\mathrm{E}\left(N_{i}\right)=L_{i}(t)$

$$
=\left[\lambda \theta_{i-1}\left\{\frac{1}{\mu_{i}}\left(1-e^{-\mu_{i} t}\right)+\frac{1}{\left(\mu_{i-} \mu_{1}\right)}\left(e^{-\mu_{i} t}-e^{-\mu_{1} t}\right)\right\}\right]
$$

Variation in number of customers in $\mathbf{i}^{\text {th }}$ buffer is $\operatorname{Var}\left(N_{i}\right)$

$=\left[\lambda \theta_{i-1}\left\{\frac{1}{\mu_{i}}\left(1-e^{-\mu_{i} t}\right)+\frac{1}{\left(\mu_{i-} \mu_{1}\right)}\left(e^{-\mu_{i} t}-e^{-\mu_{1} t}\right)\right\}\right]$

Putting $n_{i}=0$ in (17) we get the probability that the $\mathrm{i}^{\text {th }}$ buffer is empty as $P(., \ldots, ., 0 ; t)=\exp$

$$
\left[\left(-\lambda \theta_{i-1}\right)\left\{\frac{1}{\mu_{i}}\left(1-e^{-\mu_{i} t}\right)+\frac{1}{\left(\mu_{i-} \mu_{1}\right)}\left(e^{-\mu_{i} t}-e^{-\mu_{1} t}\right)\right\}\right]
$$

Utilization of $\mathbf{i}^{\text {th }}$ server is

$U_{i}(t)=1-P(., . ., 0 ; t)=1-\exp \left[\left(-\lambda \theta_{i}\right)\left\{\frac{1}{\mu_{i}}\left(1-e^{-\mu_{i} t}\right)+\right.\right.$ $\left.\left.\frac{1}{\left(\mu_{i-} \mu_{1}\right)}\left(e^{-\mu_{i} t}-e^{-\mu_{1} t}\right)\right\}\right]$

Throughput of $\mathbf{i}^{\text {th }} \quad$ server $\quad$ isThp $p_{i}(t)=\mu_{i} \cdot U_{i}=\mu_{i} \cdot[1-$ $\left.\exp \left[\left(-\lambda \theta_{i-1}\right)\left\{\frac{1}{\mu_{i}}\left(1-e^{-\mu_{i} t}\right)+\frac{1}{\left(\mu_{i-} \mu_{1}\right)}\left(e^{-\mu_{i} t}-e^{-\mu_{1} t}\right)\right\}\right]\right]$

Average waiting time of customers in $\mathbf{i}^{\text {th }}$ buffer (average delay in $\mathbf{i}^{\text {th }}$ server )is

$$
\begin{aligned}
& W_{i}(t)=\frac{L_{i}(t)}{\operatorname{Thp}_{i}(t)} \\
& =\frac{\left[\lambda \theta_{i-1}\left\{\frac{1}{\mu_{i}}\left(1-e^{-\mu_{i} t}\right)+\frac{1}{\left(\mu_{i-} \mu_{1}\right)}\left(e^{-\mu_{i} t}-e^{-\mu_{1} t}\right)\right\}\right]}{\mu_{i \cdot} \cdot\left[1-\exp \left[\left(-\lambda \theta_{i-1}\right)\left\{\frac{1}{\mu_{i}}\left(1-e^{-\mu_{i} t}\right)+\frac{1}{\left(\mu_{i-} \mu_{1}\right)}\left(e^{-\mu_{i} t}-e^{-\mu_{1} t}\right)\right\}\right]\right]} \\
& =1-\exp \left[\frac{-\lambda}{\mu_{1}}\left(1-e^{-\mu_{1} t}\right)\right]
\end{aligned}
$$

entire system $L(t)$ also gets decreased. Thus the improvement in performance of one server improves the performance of entire system. Similarly when $\mu_{2}$ is increased $L_{2}(\mathrm{t})$ decreases,$\mu_{3}$ is increased $L_{3}(\mathrm{t})$ decreases etc., On the same lines when the probability $\theta_{1}\left(\operatorname{or} \theta_{2}\right)$ that the customers from first server join second (or third server) increases the buffer at second server $L_{2}(t)$ (or at third server $L_{3}(t)$ ) is increasing correspondingly.

Table 2 indicates that with respect to time the probability of emptiness has shown sudden decrease initially ( $\mathrm{t}=0.1$ only) and decreasing normally thereafter(for $\mathrm{t}=0.2,0.3,0.4,0.5$ ). Similarly with increase in mean arrival rate $\lambda$ the probability of emptiness at each server is decreasing while the utilization of servers $U_{1}, U_{2}, U_{3}, U_{4}$ is increasing. This clearly indicates that the system is performing according to the requirement. As the number of customers served at each server increases $\left(\mu_{1}=10\right.$ to 14$)$ the system tends towards rest. Thus the probability of emptiness increases while utilization of servers decreases as is expected.

The probability of emptiness decreases as the probability of customers joining a particular server while it's utilization gets increased. Thus as $\theta_{1}$ increases from 0.1 to 0.5 system emptiness increases from 0.1480 to 0.3479 .This has an impact on the fourth server by increasing the probability of emptiness at fourth server from 0.8233 to 0.9200 and decreasing it's utilization from 0.1767 to 0.0800 . Similarly with increase of $\theta_{2}$ from 0.1 to 0.5 the probability of emptiness of third server decreases and it's utilization increases whereas emptiness of fourth server increases and it's utilization decreases. 
From Table. 3 it is observed that the throughputs $T h p_{1}, T h p_{2}, T h p_{3}, T h p_{4}$ and mean waiting times $W_{1}, W_{2}, W_{3}, W_{4}$ at each of the four servers have shown increase with increase in time. Similarly an increase in $\lambda$ lead to an increase in throughputs as well as mean waiting times. Further we can observe that the increase in service rate at second, third and fourth server leads to increase in throughputs and waiting times except at first server whereas increase in $\mu_{1}$ leads to increase in $T h p_{1}$ and decrease in $W_{1}$.

The probability of joining second server $\left(\mathrm{S}_{2}\right)$ increases from $\theta_{1}=0.1$ to 0.5 the throughput $T h p_{2}$ increases correspondingly from 0.2037 to 0.9610 , this in turn increase the waiting time $W_{2}$ from 0.1450 to 0.1537 . As this influences on $\theta_{3}$ which decreases from 0.7 to 0.3 ,the throughput $T h p_{4}$ decreases from 1.5907 to 0.7198 while decreasing in mean waiting time $W_{4}$ is indicated from 0.1223 to 0.1158 . Therefore the data supports the theoretical expectations. Similar changes can also be observed in case of changes in probability of joining third server $\left(S_{3}\right)$ after being served at first server $\left(\mathrm{S}_{1}\right)$.

\section{Sensitivity Analysis}

Table1: Values of Expected Number (Mean) of Customers in the Queue in Transient State.

\begin{tabular}{|c|c|c|c|c|c|c|c|c|c|c|c|c|c|}
\hline $\mathrm{t}$ & $\lambda$ & $\mu_{1}$ & $\mu_{2}$ & $\mu_{3}$ & $\mu_{4}$ & $\theta_{1}$ & $\theta_{2}$ & $\theta_{3}$ & $L_{1}(t)$ & $L_{2}(t)$ & $L_{3}(t)$ & $L_{4}(t)$ & $L(t)$ \\
\hline 0.1 & 15 & 6 & 7 & 8 & 9 & 0.1 & 0.2 & 0.7 & 1.1280 & 0.0295 & 0.0573 & 0.1945 & 1.4093 \\
\hline 0.2 & 15 & 6 & 7 & 8 & 9 & 0.1 & 0.2 & 0.7 & 1.7470 & 0.0795 & 0.1503 & 0.4982 & 2.4750 \\
\hline 0.3 & 15 & 6 & 7 & 8 & 9 & 0.1 & 0.2 & 0.7 & 2.0868 & 0.1238 & 0.2291 & 0.7449 & 3.1846 \\
\hline 0.4 & 15 & 6 & 7 & 8 & 9 & 0.1 & 0.2 & 0.7 & 2.2732 & 0.1564 & 0.2848 & 0.9129 & 3.6273 \\
\hline 0.5 & 15 & 6 & 7 & 8 & 9 & 0.1 & 0.2 & 0.7 & 2.3755 & 0.1784 & 0.3209 & 1.0183 & 3.8391 \\
\hline 0.1 & 10 & 6 & 7 & 8 & 9 & 0.1 & 0.2 & 0.7 & 0.7520 & 0.0197 & 0.0382 & 0.1297 & 0.9396 \\
\hline 0.1 & 11 & 6 & 7 & 8 & 9 & 0.1 & 0.2 & 0.7 & 0.8272 & 0.0217 & 0.0420 & 0.1426 & 1.0335 \\
\hline 0.1 & 12 & 6 & 7 & 8 & 9 & 0.1 & 0.2 & 0.7 & 0.9024 & 0.0236 & 0.0458 & 0.1556 & 1.1274 \\
\hline 0.1 & 13 & 6 & 7 & 8 & 9 & 0.1 & 0.2 & 0.7 & 0.9776 & 0.0256 & 0.0496 & 0.1686 & 1.2214 \\
\hline 0.1 & 14 & 6 & 7 & 8 & 9 & 0.1 & 0.2 & 0.7 & 1.0528 & 0.0276 & 0.0535 & 0.1815 & 1.3154 \\
\hline 0.1 & 15 & 10 & 7 & 8 & 9 & 0.1 & 0.2 & 0.7 & 0.9482 & 0.0435 & 0.0843 & 0.2861 & 1.3621 \\
\hline 0.1 & 15 & 11 & 7 & 8 & 9 & 0.1 & 0.2 & 0.7 & 0.9097 & 0.0465 & 0.0900 & 0.3054 & 1.3516 \\
\hline 0.1 & 15 & 12 & 7 & 8 & 9 & 0.1 & 0.2 & 0.7 & 0.8735 & 0.0493 & 0.0954 & 0.3235 & 1.3417 \\
\hline 0.1 & 15 & 13 & 7 & 8 & 9 & 0.1 & 0.2 & 0.7 & 0.8394 & 0.0519 & 0.1004 & 0.3405 & 1.3322 \\
\hline 0.1 & 15 & 14 & 7 & 8 & 9 & 0.1 & 0.2 & 0.7 & 0.8072 & 0.0543 & 0.1051 & 0.3564 & 1.3230 \\
\hline 0.1 & 15 & 6 & 10 & 8 & 9 & 0.1 & 0.2 & 0.7 & 1.1280 & 0.0270 & 0.0573 & 0.1945 & 1.4068 \\
\hline 0.1 & 15 & 6 & 11 & 8 & 9 & 0.1 & 0.2 & 0.7 & 1.1280 & 0.0262 & 0.0573 & 0.1945 & 1.4060 \\
\hline 0.1 & 15 & 6 & 12 & 8 & 9 & 0.1 & 0.2 & 0.7 & 1.1280 & 0.0254 & 0.0573 & 0.1945 & $\begin{array}{l}1.4052 \\
1.4045\end{array}$ \\
\hline 0.1 & 15 & 6 & 13 & 8 & 9 & 0.1 & 0.2 & 0.7 & 1.1280 & 0.0247 & 0.0573 & 0.1945 & 1.4045 \\
\hline 0.1 & 15 & 6 & 14 & 8 & 9 & 0.1 & 0.2 & 0.7 & 1.1280 & 0.0241 & 0.0573 & 0.1945 & \\
\hline 0.1 & 15 & 6 & 7 & 10 & 9 & 0.1 & 0.2 & 0.7 & 1.1280 & 0.0295 & 0.0539 & 0.1945 & 1.4059 \\
\hline 0.1 & 15 & 6 & 7 & 11 & 9 & 0.1 & 0.2 & 0.7 & 1.1280 & 0.0295 & 0.0524 & 0.1945 & 1.4044 \\
\hline 0.1 & 15 & 6 & 7 & 12 & 9 & 0.1 & 0.2 & 0.7 & 1.1280 & 0.0295 & 0.0509 & 0.1945 & 1.4015 \\
\hline 0.1 & 15 & 6 & 7 & 13 & 9 & 0.1 & 0.2 & 0.7 & 1.1280 & 0.0295 & 0.0495 & 0.1945 & 1.4029 \\
\hline 0.1 & 15 & 6 & 7 & 14 & 9 & 0.1 & 0.2 & 0.7 & 1.1280 & 0.0295 & 0.0481 & 0.1945 & 1.4001 \\
\hline 0.1 & 15 & 6 & 7 & 8 & 10 & 0.1 & 0.2 & 0.7 & 1.1280 & 0.0295 & 0.0573 & 0.1888 & 1.4036 \\
\hline 0.1 & 15 & 6 & 7 & 8 & 11 & 0.1 & 0.2 & 0.7 & 1.1280 & 0.0295 & 0.0573 & 0.1833 & 1.3981 \\
\hline 0.1 & 15 & 6 & 7 & 8 & 12 & 0.1 & 0.2 & 0.7 & 1.1280 & 0.0295 & 0.0573 & 0.1781 & 1.3929 \\
\hline 0.1 & 15 & 6 & 7 & 8 & 13 & 0.1 & 0.2 & 0.7 & 1.1280 & 0.0295 & 0.0573 & 0.1732 & 1.3880 \\
\hline 0.1 & 15 & 6 & 7 & 8 & 14 & 0.1 & 0.2 & 0.7 & 1.1280 & 0.0295 & 0.0573 & 0.1684 & 1.3832 \\
\hline 0.1 & 15 & 6 & 7 & 8 & 9 & 0.1 & 0.2 & 0.7 & 1.1280 & 0.0295 & 0.0573 & 0.1945 & 1.4093 \\
\hline 0.1 & 15 & 6 & 7 & 8 & 9 & 0.2 & 0.2 & 0.6 & 1.1280 & 0.0591 & 0.0573 & 0.1667 & 1.3815 \\
\hline 0.1 & 15 & 6 & 7 & 8 & 9 & 0.3 & 0.2 & 0.5 & 1.1280 & 0.0886 & 0.0573 & 0.1389 & 1.3537 \\
\hline 0.1 & 15 & 6 & 7 & 8 & 9 & 0.4 & 0.2 & 0.4 & 1.1280 & 0.1181 & 0.0573 & 0.1111 & 1.3259 \\
\hline 0.1 & 15 & 6 & 7 & 8 & 9 & 0.5 & 0.2 & 0.3 & 1.1280 & 0.1477 & 0.0573 & 0.0834 & 1.2982 \\
\hline 0.1 & 15 & 6 & 7 & 8 & 9 & 0.1 & 0.1 & 0.8 & 1.1280 & 0.0295 & 0.0286 & 0.2223 & 1.4084 \\
\hline 0.1 & 15 & 6 & 7 & 8 & 9 & 0.1 & 0.2 & 0.7 & 1.1280 & 0.0295 & 0.0573 & 0.1945 & 1.4389 \\
\hline 0.1 & 15 & 6 & 7 & 8 & 9 & 0.1 & 0.3 & 0.6 & 1.1280 & 0.0295 & 0.0859 & 0.1667 & 1.4692 \\
\hline 0.1 & 15 & 6 & 7 & 8 & 9 & 0.1 & 0.4 & 0.5 & 1.1280 & 0.0295 & 0.1146 & 0.1389 & 1.4996 \\
\hline 0.1 & 15 & 6 & 7 & 8 & 9 & 0.1 & 0.5 & 0.4 & 1.1280 & 0.0295 & 0.1432 & 0.1111 & 1.5300 \\
\hline 0.1 & 15 & 6 & 7 & 8 & 9 & 0.1 & 0.2 & 0.7 & 1.1280 & 0.0295 & 0.0573 & 0.1945 & 1.4093 \\
\hline 0.1 & 15 & 6 & 7 & 8 & 9 & 0.1 & 0.2 & 0.7 & 1.1280 & 0.0295 & 0.0573 & 0.1945 & 1.4093 \\
\hline 0.1 & 15 & 6 & 7 & 8 & 9 & 0.1 & 0.2 & 0.7 & 1.1280 & 0.0295 & 0.0573 & 0.1945 & $\begin{array}{l}1.4093 \\
14093\end{array}$ \\
\hline 0.1 & 15 & 6 & 7 & 8 & 9 & 0.1 & 0.2 & 0.7 & 1.1280 & 0.0295 & 0.0573 & 0.1945 & $\begin{array}{l}1.4093 \\
1.43\end{array}$ \\
\hline 0.1 & 15 & 6 & 7 & 8 & 9 & 0.1 & 0.2 & 0.7 & 1.1280 & 0.0295 & 0.0573 & 0.1945 & 1.4093 \\
\hline
\end{tabular}
the values of parameters as $\mathrm{t}=0.1$, $\lambda=15, \mu_{1}=12, \mu_{2}=14, \mu_{3}=11, \mu_{4}=13, \theta_{1}=0.3 \quad$ and $\quad \theta_{2}=0.2$.The effect of variation of $\mp 15 \%, \mp 10 \%$ and $\mp 5 \%$ on the performance measures $\mathrm{L}_{1}, \mathrm{~L}_{2}, \mathrm{~L}_{3}, \mathrm{~L}_{4}, \mathrm{~L}, \mathrm{~W}_{1}, \mathrm{~W}_{2}, \mathrm{~W}_{3}$ and $\mathrm{W}_{4}$ were computed and are given in Table 4.

From Table 4.it is observed that as time $\mathrm{t}$ increases all the values of $\mathrm{L}_{1}, \mathrm{~L}_{2}, \mathrm{~L}_{3}, \mathrm{~L}_{4}, \mathrm{~L}, \mathrm{~W}_{1}, \mathrm{~W}_{2}, \mathrm{~W}_{3}$ and $\mathrm{W}_{4}$ increase and decrease when time decreases. We can observe the same phenomenon with variation in arrival rate $\lambda$.It is also observed that as $\mu_{1}$ increases $\mathrm{L}_{1}, \mathrm{~L}$ and $\mathrm{W}_{1}$ are decreasing are decreasing whereas $\mathrm{L}_{1}, \mathrm{~L}_{2}, \mathrm{~L}_{4}, \mathrm{~W}_{1}, \mathrm{~W}_{2}$ and $\mathrm{W}_{4}$ are constants. As $\mu_{3}$ increases $\mathrm{L}_{3}, \mathrm{~L}, \mathrm{~W}_{3}$ are decreasing whereas $\mathrm{L}_{1}, \mathrm{~L}_{2}, \mathrm{~L}_{4}, \mathrm{~W}_{1}, \mathrm{~W}_{2}$ and $\mathrm{W}_{4}$ are constants and as $\mu_{4}$ increases $L_{4}, L, W_{4}$ are decreasing whereas $\mathrm{L}_{1}, \mathrm{~L}_{2}, \mathrm{~L}_{3}, \mathrm{~L}_{4}, \mathrm{~W}_{1}, \mathrm{~W}_{2}, \mathrm{~W}_{3}$ and $\mathrm{W}_{4}$ are constants.

It is also observed that as $\theta_{1}$ increases $L_{2}, L, W_{2}$ are increasing, $\mathrm{L}_{4}, \mathrm{~W}_{4}$ are decreasing whereas $\mathrm{L}_{1}, \mathrm{~L}_{3}, \mathrm{~W}_{1}$ and $\mathrm{W}_{3}$ are constants .Similarly as $\theta_{3}$ increases $L_{3}, L, W_{3}$ are increasing, $L_{4}$ and $W_{4}$ are decreasing and $\mathrm{L}_{1}, \mathrm{~L}_{2}, \mathrm{~W}_{1}$ and $\mathrm{W}_{2}$ are constants. 
Table 2: Probability of Emptiness and Utilization of Servers and System in Transient State.

\begin{tabular}{|c|c|c|c|c|c|c|c|c|c|c|c|c|c|c|c|c|c|}
\hline$t$ & $\lambda$ & $\mu_{1}$ & $\mu_{2}$ & $\mu_{3}$ & $\mu_{4}$ & $\theta_{1}$ & $\theta_{2}$ & $\theta_{3}$ & $P_{0000} t$ & $P_{0 \ldots} t$ & $P_{.0 . .} t$ & $P_{.0 .} t$ & $P_{\ldots 0} t$ & $U_{1}(t)$ & $U_{2}(t)$ & $U_{3}(t)$ & $U_{4}(t)$ \\
\hline 0.1 & 15 & 6 & 7 & 8 & 9 & 0.1 & 0.2 & 0.7 & 0.2443 & 0.3237 & 0.9709 & 0.9443 & 0.8233 & 0.6763 & 0.0291 & 0.0557 & 0.1767 \\
\hline 0.2 & 15 & 6 & 7 & 8 & 9 & 0.1 & 0.2 & 0.7 & 0.0842 & 0.1743 & 0.9235 & 0.8604 & 0.6076 & 0.8257 & 0.0765 & 0.1396 & 0.3924 \\
\hline 0.3 & 15 & 6 & 7 & 8 & 9 & 0.1 & 0.2 & 0.7 & 0.0414 & 0.1241 & 0.8836 & 0.7952 & 0.4748 & 0.8759 & 0.1164 & 0.2048 & 0.5252 \\
\hline 0.4 & 15 & 6 & 7 & 8 & 9 & 0.1 & 0.2 & 0.7 & 0.0266 & 0.1030 & 0.8552 & 0.7522 & 0.4014 & 0.8970 & 0.1448 & 0.2478 & 0.5986 \\
\hline 0.5 & 15 & 6 & 7 & 8 & 9 & 0.1 & 0.2 & 0.7 & 0.0204 & 0.0930 & 0.8366 & 0.7255 & 0.3612 & 0.9070 & 0.1634 & 0.2745 & 0.6388 \\
\hline 0.1 & 10 & 6 & 7 & 8 & 9 & 0.1 & 0.2 & 0.7 & 0.3908 & 0.4714 & 0.9805 & 0.9625 & 0.8784 & 0.5266 & 0.0195 & 0.0375 & 0.1216 \\
\hline 0.1 & 11 & 6 & 7 & 8 & 9 & 0.1 & 0.2 & 0.7 & 0.3558 & 0.4373 & 0.9786 & 0.9589 & 0.8671 & 0.5627 & 0.0214 & 0.0411 & 0.1329 \\
\hline 0.1 & 12 & 6 & 7 & 8 & 9 & 0.1 & 0.2 & 0.7 & 0.3239 & 0.4056 & 0.9766 & 0.9552 & 0.8559 & 0.5944 & 0.0234 & 0.0448 & 0.1441 \\
\hline 0.1 & 14 & 6 & 7 & 8 & 9 & 0.1 & 0.2 & 0.7 & 0.2684 & 0.3490 & 0.9728 & 0.9479 & 0.8340 & 0.6510 & 0.0272 & 0.0521 & 0.1660 \\
\hline 0.1 & 15 & 10 & 7 & 8 & 9 & 0.1 & 0.2 & 0.7 & 0.2561 & 0.3874 & 0.9574 & 0.9191 & 0.7512 & & 0.0426 & 0.0809 & 0.2468 \\
\hline 0.1 & 15 & 11 & 7 & 8 & 9 & 0.1 & 0.2 & 0.7 & 0.2588 & 0.4026 & 0.9546 & 0.9139 & 0.7368 & & 0.0454 & 0.0861 & 0.2632 \\
\hline 0.1 & 15 & 12 & 7 & 8 & 9 & 0.1 & 0.2 & 0.7 & 0.2614 & 0.4175 & 0.9519 & 0.9090 & 0.7236 & & 0.0481 & 0.0910 & 0.2764 \\
\hline 0.1 & 15 & 13 & 7 & 8 & 9 & 0.1 & 0.2 & 0.7 & 0.2639 & 0.4320 & 0.9495 & 0.9045 & 0.7114 & & 0.0505 & 0.0955 & 0.2886 \\
\hline 0.1 & 15 & 14 & 7 & 8 & 9 & 0.1 & 0.2 & 0.7 & 0.2663 & 0.4461 & 0.9471 & 0.9002 & 0.7002 & & 0.0529 & 0.0998 & 0.2998 \\
\hline 0.1 & 15 & 6 & 10 & 8 & 9 & 0.1 & 0.2 & 0.7 & 0.2449 & 0.3237 & 0.9734 & 0.9443 & 0.8233 & 0.6763 & 0.0266 & 0.0557 & 0.1767 \\
\hline 0.1 & 15 & 6 & 11 & 8 & 9 & 0.1 & 0.2 & 0.7 & 0.2451 & 0.3237 & 0.9742 & 0.9443 & 0.8233 & 0.6763 & 0.0258 & 0.0557 & 0.1767 \\
\hline 0.1 & 15 & 6 & 13 & 8 & 9 & 0.1 & 0.2 & 0.7 & 0.2455 & 0.3237 & 0.9756 & 0.9443 & 0.8233 & 0.6763 & 0.0244 & 0.0557 & 0.1767 \\
\hline 0.1 & 15 & 6 & 14 & 8 & 9 & 0.1 & 0.2 & 0.7 & 0.2457 & 0.3237 & 0.9762 & 0.9443 & 0.8233 & 0.6763 & 0.0238 & 0.0557 & 0.1767 \\
\hline 0.1 & 15 & 6 & 7 & 10 & 9 & 0.1 & 0.2 & 0.7 & 0.2451 & 0.3237 & 0.9709 & 0.9475 & 0.8233 & 0.6763 & 0.0291 & 0.0525 & 0.1767 \\
\hline 0.1 & 15 & 6 & 7 & 11 & 9 & 0.1 & 0.2 & 0.7 & 0.2455 & 0.3237 & 0.9709 & 0.9490 & 0.8233 & 0.6763 & 0.0291 & 0.0510 & 0.1767 \\
\hline 0.1 & 15 & 6 & 7 & 12 & 9 & 0.1 & 0.2 & 0.7 & 0.2459 & 0.3237 & 0.9709 & 0.9504 & 0.8233 & 0.6763 & 0.0291 & 0.0496 & 0.1767 \\
\hline 0.1 & 15 & 6 & 7 & 13 & 9 & 0.1 & 0.2 & 0.7 & 0.2462 & 0.3237 & 0.9709 & 0.9517 & 0.8233 & 0.6763 & 0.0291 & 0.0483 & 0.1767 \\
\hline 0.1 & 15 & 6 & 7 & 14 & 9 & 0.1 & 0.2 & 0.7 & 0.2466 & 0.3237 & 0.9709 & 0.9530 & 0.8233 & 0.6763 & 0.0291 & 0.0470 & 0.1767 \\
\hline 0.1 & 15 & 6 & 7 & 8 & 10 & 0.1 & 0.2 & 0.7 & 0.2457 & 0.3237 & 0.9709 & 0.9443 & 0.8280 & 0.6763 & 0.0291 & 0.0557 & 0.1720 \\
\hline 0.1 & 15 & 6 & 7 & 8 & 11 & 0.1 & 0.2 & 0.7 & 0.2471 & 0.3237 & 0.9709 & 0.9443 & 0.8325 & 0.6763 & 0.0291 & 0.0557 & 0.1675 \\
\hline 0.1 & 15 & 6 & 7 & 8 & 12 & 0.1 & 0.2 & 0.7 & 0.2484 & 0.3237 & 0.9709 & 0.9443 & 0.8368 & 0.6763 & 0.0291 & 0.0557 & 0.1632 \\
\hline 0.1 & 15 & 6 & 7 & 8 & 13 & 0.1 & 0.2 & 0.7 & 0.2496 & 0.3237 & 0.9709 & 0.9443 & 0.8410 & 0.6763 & 0.0291 & 0.0557 & 0.1590 \\
\hline 0.1 & 15 & 6 & 7 & 8 & 14 & 0.1 & 0.2 & 0.7 & 0.2508 & 0.3237 & 0.9709 & 0.9443 & 0.8450 & 0.6763 & 0.0291 & 0.0557 & 0.1550 \\
\hline 0.1 & 15 & 6 & 7 & 8 & 9 & 0.1 & 0.2 & 0.7 & 0.1480 & 0.3237 & 0.9709 & 0.9443 & 0.8233 & 0.6763 & 0.0291 & 0.0557 & 0.1767 \\
\hline 0.1 & 15 & 6 & 7 & 8 & 9 & 0.2 & 0.2 & 0.6 & 0.1835 & 0.3237 & 0.9426 & 0.9443 & 0.8464 & 0.6763 & 0.0574 & 0.0557 & 0.1536 \\
\hline 0.1 & 15 & 6 & 7 & 8 & 9 & 0.3 & 0.2 & 0.5 & 0.2275 & 0.3237 & 0.9152 & 0.9443 & 0.8703 & 0.6763 & 0.0848 & 0.0557 & 0.1297 \\
\hline 0.1 & 15 & 6 & 7 & 8 & 9 & 0.4 & 0.2 & 0.4 & 0.2822 & 0.3237 & 0.8886 & 0.9443 & 0.8948 & 0.6763 & 0.1114 & 0.0557 & 0.1052 \\
\hline 0.1 & 15 & 6 & 7 & 8 & 9 & 0.5 & 0.2 & 0.3 & 0.3499 & 0.3237 & 0.8627 & 0.9443 & 0.9200 & 0.6763 & 0.1373 & 0.0557 & 0.0800 \\
\hline 0.1 & 15 & 6 & 7 & 8 & 9 & 0.1 & 0.3 & 0.6 & 0.2441 & 0.3237 & 0.9709 & 0.9177 & 0.8464 & 0.6763 & 0.0291 & 0.0823 & 0.1536 \\
\hline 0.1 & 15 & 6 & 7 & 8 & 9 & 0.1 & 0.4 & 0.5 & 0.2439 & 0.3237 & 0.9709 & 0.8918 & 0.8703 & 0.6763 & 0.0291 & 0.1082 & 0.1297 \\
\hline 0.1 & 15 & 6 & 7 & 8 & 9 & 0.1 & 0.5 & 0.4 & 0.2437 & 0.3237 & 0.9709 & 0.8666 & 0.8948 & 0.6763 & 0.0291 & 0.1334 & 0.1052 \\
\hline 0.1 & 15 & 6 & 7 & 8 & 9 & 0.1 & 0.2 & 0.7 & 0.2443 & 0.3237 & 0.9709 & 0.9443 & 0.8233 & 0.6763 & 0.0291 & 0.0557 & 0.1767 \\
\hline 0.1 & 15 & 6 & 7 & 8 & 9 & 0.1 & 0.2 & 0.7 & 0.2443 & 0.3237 & 0.9709 & 0.9443 & 0.8233 & 0.6763 & 0.0291 & 0.0557 & 0.1767 \\
\hline 0.1 & 15 & 6 & 7 & 8 & 9 & 0.1 & 0.2 & 0.7 & 0.2443 & 0.3237 & 0.9709 & 0.9443 & 0.8233 & 0.6763 & 0.0291 & 0.0557 & 0.1767 \\
\hline 0.1 & 15 & 6 & 7 & 8 & 9 & 0.1 & 0.2 & 0.7 & 0.2443 & 0.3237 & 0.9709 & 0.9443 & 0.8233 & 0.6763 & 0.0291 & 0.0557 & 0.1767 \\
\hline 0.1 & 15 & 6 & 7 & 8 & 9 & 0.1 & 0.2 & 0.7 & 0.2443 & 0.3237 & 0.9709 & 0.9443 & 0.8233 & 0.6763 & 0.0291 & 0.0557 & 0.1767 \\
\hline
\end{tabular}

Table 3: Values of Throughput and Waiting Time of Customers in Queues in Transient State.

\begin{tabular}{|c|c|c|c|c|c|c|c|c|c|c|c|c|c|c|c|c|}
\hline$t$ & $\lambda$ & $\mu_{1}$ & $\mu_{2}$ & $\mu_{3}$ & $\mu_{4}$ & $\theta_{1}$ & $\boldsymbol{\theta}_{2}$ & $\boldsymbol{\theta}_{3}$ & $T h p_{1}(t)$ & $T h p_{2}(t)$ & $T h p_{3}(t)$ & $T h p_{4}(t)$ & $W_{1}(t)$ & $W_{2}(t)$ & $W_{3}(t)$ & $W_{4}(t)$ \\
\hline 0.1 & 15 & 6 & 7 & 8 & 9 & 0.1 & 0.2 & 0.7 & 4.0579 & 0.2037 & 0.4453 & 1.5907 & 0.2780 & 0.1450 & 0.1286 & 0.1223 \\
\hline 0.2 & 15 & 6 & 7 & 8 & 9 & 0.1 & 0.2 & 0.7 & 4.9542 & 0.5353 & 1.1167 & 3.5313 & 0.3526 & 0.1486 & 0.1346 & 0.1411 \\
\hline 0.3 & 15 & 6 & 7 & 8 & 9 & 0.1 & 0.2 & 0.7 & 5.2555 & 0.8150 & 1.6381 & 4.7271 & 0.3971 & 0.1519 & 0.1399 & 0.1576 \\
\hline 0.4 & 15 & 6 & 7 & 8 & 9 & 0.1 & 0.2 & 0.7 & 5.3821 & 1.0134 & 1.9826 & 5.3878 & 0.4224 & 0.1543 & 0.1436 & 0.1694 \\
\hline 0.5 & 15 & 6 & 7 & 8 & 9 & 0.1 & 0.2 & 0.7 & 5.4422 & 1.1439 & 2.1962 & 5.7492 & 0.4365 & 0.1560 & 0.1461 & 0.1771 \\
\hline 0.1 & 10 & 6 & 7 & 8 & 9 & 0.1 & 0.2 & 0.7 & 3.1714 & 0.1365 & 0.2997 & 1.0944 & 0.2371 & 0.1443 & 0.1274 & 0.1185 \\
\hline 0.1 & 11 & 6 & 7 & 8 & 9 & 0.1 & 0.2 & 0.7 & 3.3763 & 0.1500 & 0.3291 & 1.1963 & 0.2450 & 0.1444 & 0.1276 & 0.1192 \\
\hline 0.1 & 12 & 6 & 7 & 8 & 9 & 0.1 & 0.2 & 0.7 & 3.5664 & 0.1635 & 0.3583 & 1.2968 & 0.2530 & 0.1446 & 0.1279 & 0.1200 \\
\hline 0.1 & 13 & 6 & 7 & 8 & 9 & 0.1 & 0.2 & 0.7 & 3.7427 & 0.1769 & 0.3874 & 1.3960 & 0.2612 & 0.1447 & 0.1281 & 0.1207 \\
\hline 0.1 & 14 & 6 & 7 & 8 & 9 & 0.1 & 0.2 & 0.7 & 3.9062 & 0.1903 & 0.4164 & 1.4940 & 0.2695 & 0.1448 & 0.1284 & 0.1215 \\
\hline 0.1 & 15 & 10 & 7 & 8 & 9 & 0.1 & 0.2 & 0.7 & 6.1255 & 0.2981 & & 2.2392 & 0.1548 & 0.1460 & 0.1303 & 0.1278 \\
\hline 0.1 & 15 & 11 & 7 & 8 & 9 & 0.1 & 0.2 & 0.7 & 6.5750 & 0.3179 & 6889 & 2.3687 & 0.1384 & 0.1462 & 0.1307 & 0.1289 \\
\hline 0.1 & 15 & 12 & 7 & 8 & 9 & 0.1 & 0.2 & 0.7 & 6.9902 & 0.3364 & & 2.4876 & 0.1250 & 0.1464 & 0.1311 & 0.1301 \\
\hline 0.1 & 15 & 13 & 7 & 8 & 9 & 0.1 & 0.2 & 0.7 & 7.3843 & 0.3538 & & 2.5972 & 0.1137 & 0.1466 & 0.1314 & 0.1311 \\
\hline 0.1 & 15 & 14 & 7 & 8 & 9 & 0.1 & 0.2 & 0.7 & 7.7546 & 0.3700 & & 2.6982 & 0.1041 & 0.1468 & 0.1317 & 0.1321 \\
\hline 0.1 & 15 & 6 & 10 & 8 & 9 & 0.1 & 0.2 & 0.7 & 4.0579 & 0.2661 & 0.4453 & 1.5907 & 0.2780 & 0.1014 & 0.1286 & 0.1223 \\
\hline 0.1 & 15 & 6 & 11 & 8 & 9 & 0.1 & 0.2 & 0.7 & 4.0579 & 0.2843 & 0.4453 & 1.5907 & 0.2780 & 0.0921 & 0.1286 & 0.1223 \\
\hline 0.1 & 15 & 6 & 12 & 8 & 9 & 0.1 & 0.2 & 0.7 & 4.0579 & 0.3015 & 0.4453 & 1.5907 & 0.2780 & 0.0844 & 0.1286 & 0.1223 \\
\hline 0.1 & 15 & 6 & 13 & 8 & 9 & 0.1 & 0.2 & 0.7 & 4.0579 & 0.3176 & 0.4453 & 1.5907 & 0.2780 & 0.0779 & 0.1286 & 0.1223 \\
\hline 0.1 & 15 & 6 & 14 & 8 & 9 & 0.1 & 0.2 & 0.7 & 4.0579 & 0.3328 & 0.4453 & 1.5907 & 0.2780 & 0.0723 & 0.1286 & 0.1223 \\
\hline 0.1 & 15 & 6 & 7 & 10 & 9 & 0.1 & 0.2 & 0.7 & 4.0579 & 0.2037 & 0.5251 & 1.5907 & 0.2780 & 0.1450 & 0.1027 & 0.1223 \\
\hline 0.1 & 15 & 6 & 7 & 11 & 9 & 0.1 & 0.2 & 0.7 & 4.0579 & 0.2037 & 0.5613 & 1.5907 & 0.2780 & 0.1450 & 0.0933 & 0.1223 \\
\hline 0.1 & 15 & 6 & 7 & 12 & 9 & 0.1 & 0.2 & 0.7 & 4.0579 & 0.2037 & 0.5954 & 1.5907 & 0.2780 & 0.1450 & 0.0855 & 0.1223 \\
\hline 0.1 & 15 & 6 & 7 & 13 & 9 & 0.1 & 0.2 & 0.7 & 4.0579 & 0.2037 & 0.6275 & 1.5907 & 0.2780 & 0.1450 & 0.0788 & 0.1223 \\
\hline 0.1 & 15 & 6 & 7 & 14 & 9 & 0.1 & 0.2 & 0.7 & 4.0579 & 0.2037 & 0.6576 & 1.5907 & 0.2780 & 0.1450 & 0.0732 & 0.1223 \\
\hline
\end{tabular}




\begin{tabular}{|c|c|c|c|c|c|c|c|c|c|c|c|c|c|c|c|c|}
\hline 0.1 & 15 & 6 & 7 & 8 & 10 & 0.1 & 0.2 & 0.7 & 4.0579 & 0.2037 & 0.4453 & 1.7203 & 0.2780 & 0.1450 & 0.1286 & 0.1097 \\
\hline 0.1 & 15 & 6 & 7 & 8 & 11 & 0.1 & 0.2 & 0.7 & 4.0579 & 0.2037 & 0.4453 & 1.8426 & 0.2780 & 0.1450 & 0.1286 & 0.0995 \\
\hline 0.1 & 15 & 6 & 7 & 8 & 12 & 0.1 & 0.2 & 0.7 & 4.0579 & 0.2037 & 0.4453 & 1.9579 & 0.2780 & 0.1450 & 0.1286 & 0.0910 \\
\hline 0.1 & 15 & 6 & 7 & 8 & 13 & 0.1 & 0.2 & 0.7 & 4.0579 & 0.2037 & 0.4453 & 2.0669 & 0.2780 & 0.1450 & 0.1286 & 0.0838 \\
\hline 0.1 & 15 & 6 & 7 & 8 & 14 & 0.1 & 0.2 & 0.7 & 4.0579 & 0.2037 & 0.4453 & 2.1697 & 0.2780 & 0.1450 & 0.1286 & 0.0776 \\
\hline 0.1 & 15 & 6 & 7 & 8 & 9 & 0.1 & 0.2 & 0.7 & 4.0579 & 0.2037 & 0.4453 & 1.5907 & 0.2780 & 0.1450 & 0.1286 & 0.1223 \\
\hline 0.1 & 15 & 6 & 7 & 8 & 9 & 0.2 & 0.2 & 0.6 & 4.0579 & 0.4015 & 0.4453 & 1.3820 & 0.2780 & 0.1471 & 0.1286 & 0.1206 \\
\hline 0.1 & 15 & 6 & 7 & 8 & 9 & 0.3 & 0.2 & 0.5 & 4.0579 & 0.5936 & 0.4453 & 1.1673 & 0.2780 & 0.1493 & 0.1286 & 0.1190 \\
\hline 0.1 & 15 & 6 & 7 & 8 & 9 & 0.4 & 0.2 & 0.4 & 4.0579 & 0.7800 & 0.4453 & 0.9466 & 0.2780 & 0.1515 & 0.1286 & 0.1174 \\
\hline 0.1 & 15 & 6 & 7 & 8 & 9 & 0.1 & 0.1 & 0.8 & 4.0579 & 0.2037 & 0.2259 & 1.7937 & 0.2780 & 0.1450 & 0.1268 & 0.1239 \\
\hline 0.1 & 15 & 6 & 7 & 8 & 9 & 0.1 & 0.2 & 0.7 & 4.0579 & 0.2037 & 0.4453 & 1.5907 & 0.2780 & 0.1450 & 0.1286 & 0.1233 \\
\hline 0.1 & 15 & 6 & 7 & 8 & 9 & 0.1 & 0.3 & 0.6 & 4.0579 & 0.2037 & 0.6586 & 1.3820 & 0.2780 & 0.1450 & 0.1304 & 0.1206 \\
\hline 0.1 & 15 & 6 & 7 & 8 & 9 & 0.1 & 0.4 & 0.5 & 4.0579 & 0.2037 & 0.8659 & 1.1673 & 0.2780 & 0.1450 & 0.1323 & 0.1190 \\
\hline 0.1 & 15 & 6 & 7 & 8 & 9 & 0.1 & 0.5 & 0.4 & 4.0579 & 0.2037 & 1.0673 & 0.9466 & 0.2780 & 0.1450 & 0.1342 & 0.1174 \\
\hline 0.1 & 15 & 6 & 7 & 8 & 9 & 0.1 & 0.2 & 0.7 & 4.0579 & 0.2037 & 0.4453 & 1.5907 & 0.2780 & 0.1450 & 0.1286 & 0.1223 \\
\hline 0.1 & 15 & 6 & 7 & 8 & 9 & 0.1 & 0.2 & 0.7 & 4.0579 & 0.2037 & 0.4453 & 1.5907 & 0.2780 & 0.1450 & 0.1286 & 0.1223 \\
\hline 0.1 & 15 & 6 & 7 & 8 & 9 & 0.1 & 0.2 & 0.7 & 4.0579 & 0.2037 & 0.4453 & 1.5907 & 0.2780 & 0.1450 & 0.1286 & 0.1223 \\
\hline 0.1 & 15 & 6 & 7 & 8 & 9 & 0.1 & 0.2 & 0.7 & 4.0579 & 0.2037 & 0.4453 & 1.5907 & 0.2780 & 0.1450 & 0.1286 & 0.1223 \\
\hline
\end{tabular}

Table 4:. Values of $L_{1}, L_{2}, L_{3}, L_{4}, L, W_{1}, W_{2}, W_{3}$ and $W_{4}$ for different Values of $t, \lambda, \mu_{1}, \mu_{2}, \mu_{3}, \mu_{4}, \theta_{1}, \theta_{2}$ and $\theta_{3}$.(SENSITIVITY ANALYSIS)

\begin{tabular}{|c|c|c|c|c|c|c|c|c|}
\hline \multirow{2}{*}{$\begin{array}{l}\text { Variation } \\
\text { Parameter }\end{array}$} & \multirow{2}{*}{$\begin{array}{l}\text { Performance } \\
\text { Measure }\end{array}$} & \multicolumn{7}{|c|}{ Percentage Change in Parameter } \\
\hline & & $-15 \%$ & $-10 \%$ & $-5 \%$ & $\mathbf{0}$ & $5 \%$ & $10 \%$ & $15 \%$ \\
\hline \multirow{8}{*}{$\mathbf{t}=\mathbf{0 . 1}$} & $\mathrm{L}_{1}(\mathrm{t})$ & 0.9988 & 1.0431 & 1.0862 & 1.1280 & 1.1685 & 1.2079 & 1.2461 \\
\hline & $\mathrm{L}_{2}(\mathrm{t})$ & 0.0227 & 0.0249 & 0.0272 & 0.0295 & 0.0319 & 0.0343 & 0.0367 \\
\hline & $\mathrm{L}_{4}(\mathrm{t})$ & 0.1507 & 0.1650 & 0.1797 & 0.1945 & 0.2095 & 0.2247 & 0.2400 \\
\hline & $\mathrm{L}(\mathrm{t})$ & 1.2164 & 1.2815 & 1.3459 & 1.4093 & 1.4717 & 1.5333 & 1.5938 \\
\hline & $\mathrm{W}_{1}(\mathrm{t})$ & 0.2635 & 0.2684 & 0.2733 & 0.2780 & 0.2826 & 0.2871 & 0.2915 \\
\hline & $\mathrm{W}_{2}(\mathrm{t})$ & 0.1445 & 0.1446 & 0.1448 & 0.1450 & 0.1451 & 0.1453 & 0.1455 \\
\hline & $\mathrm{W}_{3}(\mathrm{t})$ & 0.1278 & 0.1281 & 0.1283 & 0.1286 & 0.1289 & 0.1292 & 0.1295 \\
\hline & $W_{4}(t)$ & 0.1197 & 0.1205 & 0.1214 & 0.1223 & 0.1232 & 0.1241 & 0.1250 \\
\hline \multirow{8}{*}{$\lambda=15$} & $\mathrm{~L}_{1}(\mathrm{t})$ & 1.4850 & 1.5723 & 1.6597 & 1.7470 & 1.8344 & 1.9217 & 2.0091 \\
\hline & $\mathrm{L}_{2}(\mathrm{t})$ & 0.0676 & 0.0716 & 0.0756 & 0.0795 & 0.0835 & 0.0875 & 0.0915 \\
\hline & $\mathrm{L}_{3}(\mathrm{t})$ & 0.1278 & 0.1353 & 0.1428 & 0.1503 & 0.1579 & 0.1654 & 0.1729 \\
\hline & $\mathrm{L}_{4}(\mathrm{t})$ & 0.4235 & 0.4484 & 0.4733 & 0.4982 & 0.5231 & 0.5480 & 0.5729 \\
\hline & $\mathrm{L}(\mathrm{t})$ & 2.1039 & 2.2276 & 2.3514 & 2.475 & 2.5989 & 2.7226 & 2.8464 \\
\hline & $\mathrm{W}_{1}(\mathrm{t})$ & 0.3200 & 0.3307 & 0.3416 & 0.3526 & 0.3638 & 0.3752 & 0.3867 \\
\hline & $W_{3}(t)$ & 0.1332 & 0.1336 & 0.1341 & 0.1346 & 0.1351 & 0.1356 & 0.1361 \\
\hline & $W_{4}(t)$ & 0.1363 & 0.1379 & 0.1395 & 0.1411 & 0.1427 & 0.1443 & 0.1460 \\
\hline \multirow{9}{*}{$\mu_{1}=12$} & $\mathrm{~L}_{1}(\mathrm{t})$ & 1.8806 & 1.8345 & 1.7899 & 1.7470 & 1.7056 & 1.6656 & 1.6270 \\
\hline & $\mathrm{L}_{2}(\mathrm{t})$ & 0.0714 & 0.0743 & 0.0770 & 0.0795 & 0.0820 & 0.0844 & 0.0867 \\
\hline & $\mathrm{L}_{3}(\mathrm{t})$ & 0.1351 & 0.1404 & 0.1455 & 0.1503 & 0.1550 & 0.1595 & 0.1638 \\
\hline & $\mathrm{L}_{4}(\mathrm{t})$ & 0.4480 & 0.4655 & 0.4822 & 0.4982 & 0.5135 & 0.5283 & 0.5424 \\
\hline & $\mathrm{L}(\mathrm{t})$ & 2.5351 & 2.5147 & 2.4946 & 2.475 & 2.4561 & 2.4378 & 2.4199 \\
\hline & $W_{1}(t)$ & 0.4351 & 0.4043 & 0.3770 & 0.3526 & 0.3308 & 0.3112 & 0.2935 \\
\hline & $\mathrm{W}_{2}(\mathrm{t})$ & 0.1480 & 0.1482 & 0.1484 & 0.1486 & 0.1488 & 0.1490 & 0.1491 \\
\hline & $\mathrm{W}_{3}(\mathrm{t})$ & 0.1336 & 0.1340 & 0.1343 & 0.1346 & 0.1349 & 0.1352 & 0.1355 \\
\hline & $W_{4}(t)$ & 0.1379 & 0.1390 & 0.1400 & 0.1411 & 0.1421 & 0.1430 & 0.1440 \\
\hline \multirow{7}{*}{$\mu_{2}=14$} & $\mathrm{~L}_{1}(\mathrm{t})$ & 1.8806 & 1.8345 & 1.7899 & 1.7470 & 1.7056 & 1.6656 & 1.6270 \\
\hline & $\mathrm{L}_{2}(\mathrm{t})$ & 0.0846 & 0.0829 & 0.0812 & 0.0795 & 0.0780 & 0.0764 & 0.0750 \\
\hline & $\mathrm{L}_{3}(\mathrm{t})$ & 0.1503 & 0.1503 & 0.1503 & 0.1503 & 0.1503 & 0.1503 & 0.1503 \\
\hline & $\mathrm{L}_{4}(\mathrm{t})$ & 0.4982 & 0.4982 & 0.4982 & 0.4982 & 0.4982 & 0.4982 & 0.4982 \\
\hline & $\mathrm{L}(\mathrm{t})$ & 2.6137 & 2.5659 & 2.5196 & 2.475 & 2.4321 & 2.3905 & 2.3505 \\
\hline & $\mathrm{W}_{3}(\mathrm{t})$ & 0.1346 & 0.1346 & 0.1346 & 0.1346 & 0.1346 & 0.1346 & 0.1346 \\
\hline & $\mathrm{W}_{4}(\mathrm{t})$ & 0.1411 & 0.1411 & 0.1411 & 0.1411 & 0.1411 & 0.1411 & 0.1411 \\
\hline \multirow{9}{*}{$\mu_{3}=11$} & $\mathrm{~L}_{1}(\mathrm{t})$ & 1.7470 & 1.7470 & 1.7470 & 1.7470 & 1.7470 & 1.7470 & 1.7470 \\
\hline & $\mathrm{L}_{2}(\mathrm{t})$ & 0.0795 & 0.0795 & 0.0795 & 0.0795 & 0.0795 & 0.0795 & 0.0795 \\
\hline & $\mathrm{L}_{3}(\mathrm{t})$ & 0.1397 & 0.1357 & 0.1319 & 0.1283 & 0.1248 & 0.1215 & 0.1183 \\
\hline & $\mathrm{L}_{4}(\mathrm{t})$ & 0.4982 & 0.4982 & 0.4982 & 0.4982 & 0.4982 & 0.4982 & 0.4982 \\
\hline & $\mathrm{L}(\mathrm{t})$ & 2.4644 & 2.4604 & 2.4566 & 2.453 & 2.4495 & 2.4462 & 2.443 \\
\hline & $\mathrm{W}_{1}(\mathrm{t})$ & 0.3526 & 0.3526 & 0.3526 & 0.3526 & 0.3526 & 0.3526 & 0.3526 \\
\hline & $\mathrm{W}_{2}(\mathrm{t})$ & 0.1486 & 0.1486 & 0.1486 & 0.1486 & 0.1486 & 0.1486 & 0.1486 \\
\hline & $\mathrm{W}_{3}(\mathrm{t})$ & 0.1146 & 0.1080 & 0.1021 & 0.0969 & 0.0921 & 0.0878 & 0.0838 \\
\hline & $W_{4}(t)$ & 0.1411 & 0.1411 & 0.1411 & 0.1411 & 0.1411 & 0.1411 & 0.1411 \\
\hline \multirow{5}{*}{$\mu_{4}=13$} & $\mathrm{~L}_{1}(\mathrm{t})$ & 1.7470 & 1.7470 & 1.7470 & 1.7470 & 1.7470 & 1.7470 & 1.7470 \\
\hline & $\mathrm{L}_{2}(\mathrm{t})$ & 0.0795 & 0.0795 & 0.0795 & 0.0795 & 0.0795 & 0.0795 & 0.0795 \\
\hline & $\mathrm{L}_{3}(\mathrm{t})$ & 0.1503 & 0.1503 & 0.1503 & 0.1503 & 0.1503 & 0.1503 & 0.1503 \\
\hline & $\mathrm{L}_{4}(\mathrm{t})$ & 0.4478 & 0.4336 & 0.4201 & 0.4073 & 0.3952 & 0.3836 & 0.3727 \\
\hline & $\mathrm{L}(\mathrm{t})$ & 2.4246 & 2.4104 & 2.3969 & 2.3841 & 2.372 & 2.3604 & 2.3495 \\
\hline
\end{tabular}




\begin{tabular}{|c|c|c|c|c|c|c|c|c|}
\hline & $\mathrm{W}_{1}(\mathrm{t})$ & 0.3526 & 0.3526 & 0.3526 & 0.3526 & 0.3526 & 0.3526 & 0.3526 \\
\hline & $\mathrm{W}_{2}(\mathrm{t})$ & 0.1486 & 0.1486 & 0.1486 & 0.1486 & 0.1486 & 0.1486 & 0.1486 \\
\hline & $\mathrm{W}_{3}(\mathrm{t})$ & 0.1346 & 0.1346 & 0.1346 & 0.1346 & 0.1346 & 0.1346 & 0.1346 \\
\hline & $W_{4}(t)$ & 0.1123 & 0.1053 & 0.0992 & 0.0936 & 0.0887 & 0.0842 & 0.0801 \\
\hline \multirow{7}{*}{$\theta_{1}=0.3$} & $\mathrm{~L}_{2}(\mathrm{t})$ & 0.2028 & 0.2148 & 0.2267 & 0.2386 & 0.2506 & 0.2625 & 0.2744 \\
\hline & $\mathrm{L}_{3}(\mathrm{t})$ & 0.1503 & 0.1503 & 0.1503 & 0.1503 & 0.1503 & 0.1503 & 0.1503 \\
\hline & $\mathrm{L}_{4}(\mathrm{t})$ & 0.3879 & 0.3772 & 0.3665 & 0.3558 & 0.3452 & 0.3345 & 0.3238 \\
\hline & $W_{1}(t)$ & 0.3526 & 0.3526 & 0.3526 & 0.3526 & 0.3526 & 0.3526 & 0.3526 \\
\hline & $\mathrm{W}_{2}(\mathrm{t})$ & 0.1578 & 0.1587 & 0.1597 & 0.1606 & 0.1615 & 0.1624 & 0.1634 \\
\hline & $\mathrm{W}_{3}(\mathrm{t})$ & 0.1346 & 0.1346 & 0.1346 & 0.1346 & 0.1346 & 0.1346 & 0.1346 \\
\hline & $W_{4}(t)$ & 0.1340 & 0.1334 & 0.1327 & 0.1321 & 0.1314 & 0.1307 & 0.1301 \\
\hline \multirow{8}{*}{$\theta_{2}=0.2$} & $\mathrm{~L}_{1}(\mathrm{t})$ & 1.7470 & 1.7470 & 1.7470 & 1.7470 & 1.7470 & 1.7470 & 1.7470 \\
\hline & $\mathrm{L}_{2}(\mathrm{t})$ & 0.0795 & 0.0795 & 0.0795 & 0.0795 & 0.0795 & 0.0795 & 0.0795 \\
\hline & $\mathrm{L}_{3}(\mathrm{t})$ & 0.1278 & 0.1353 & 0.1428 & 0.1503 & 0.1579 & 0.1654 & 0.1729 \\
\hline & $\mathrm{L}_{4}(\mathrm{t})$ & 0.5195 & 0.5124 & 0.5053 & 0.4982 & 0.4911 & 0.4840 & 0.4768 \\
\hline & $W_{1}(t)$ & 0.3526 & 0.3526 & 0.3526 & 0.3526 & 0.3526 & 0.3526 & 0.3526 \\
\hline & $\mathrm{W}_{2}(\mathrm{t})$ & 0.1486 & 0.1486 & 0.1486 & 0.1486 & 0.1486 & 0.1486 & 0.1486 \\
\hline & $\mathrm{W}_{3}(\mathrm{t})$ & 0.1332 & 0.1336 & 0.1341 & 0.1346 & 0.1351 & 0.1356 & 0.1361 \\
\hline & $W_{4}(t)$ & 0.1425 & 0.1420 & 0.1415 & 0.1411 & 0.1406 & 0.1402 & 0.1397 \\
\hline
\end{tabular}

\section{Steady State Analysis}

In this section we study the steady-state analysis of queueing model by computing mean length of queue ,emptiness of server, utilization of server and average waiting time at each server.Joint Probability generating function of number of customers in first,.... $\mathrm{k}^{\text {th }}$ buffer respectively at any time $\mathrm{t}$ is

$P\left(n_{1}, n_{2}, \ldots n_{k} ; t\right)=\exp \left[\lambda\left\{\frac{1}{\mu_{1}}\left(n_{1}-1\right)\left(1-e^{-\mu_{1} t}\right)+\right.\right.$

$\frac{\theta_{1}}{\mu_{2}}\left(n_{2}-1\right)\left(1-e^{-\mu_{2} t}\right)+\cdots+\frac{\theta_{k}}{\mu_{k}}\left(n_{k}-1\right)\left(1-e^{-\mu_{k} t}\right)+$

$\frac{\theta_{1}}{\left(\mu_{2-} \mu_{1}\right)}\left(n_{2}-1\right)\left(e^{-\mu_{2} t}-e^{-\mu_{1} t}\right)+\frac{\theta_{2}}{\left(\mu_{3-} \mu_{1}\right)}\left(n_{3}-1\right)\left(e^{-\mu_{3} t}-\right.$

$\left.\left.\left.e^{-\mu_{1} t}\right)+\cdots+\frac{\theta_{k-1}}{\left(\mu_{k-} \mu_{1}\right)}\left(n_{k}-1\right)\left(e^{-\mu_{k} t}-e^{-\mu_{1} t}\right)\right\}\right]$

In steady state as $\mathrm{t} \rightarrow \infty$ we get $P\left(n_{1}, n_{2}, \ldots n_{k}\right)$

$=\exp \left[\lambda\left\{\frac{1}{\mu_{1}}\left(n_{1}-1\right)+\frac{\theta_{1}}{\mu_{2}}\left(n_{2}-1\right)+\cdots+\frac{\theta_{k-1}}{\mu_{k}}\left(n_{k}-1\right)\right\}\right](10)$

Putting $n_{1}=n_{2}=\cdots=n_{k}=0$ we get

$$
\begin{aligned}
& P(0,0, \ldots, 0)=\exp \left[\lambda\left\{-\frac{1}{\mu_{1}}-\frac{\theta_{1}}{\mu_{2}}-\cdots-\frac{\theta_{k-1}}{\mu_{k}}\right\}\right] \\
& =\exp \left[-\lambda\left\{\frac{1}{\mu_{1}}+\frac{\theta_{1}}{\mu_{2}}+\cdots+\frac{\theta_{k-1}}{\mu_{k}}\right\}\right]
\end{aligned}
$$

Which gives the probability that queue is empty in steady state

\subsection{Performance analysis of First buffer}

Putting $n_{2,}=1, n_{3,}=1, \ldots . n_{k}=1$ in $(10)$

we get probability generating function of first buffer size distribution as $P\left(n_{1}\right)=\exp \left[\lambda\left\{\frac{1}{\mu_{1}}\left(n_{1}-1\right)\right\}\right]$

Mean number of packets in first buffer is $\mathrm{E}\left(N_{1}\right)$

$=L_{1}=\frac{\lambda}{\mu_{1}}$

Variation in number of packets in first buffer is $\operatorname{Var}\left(N_{1}\right)=\frac{\lambda}{\mu_{1}}$
Putting $n_{1}=0$ in (4) we get the probability that the first buffer is empty as $P(0, ., .)=,\exp \left[\frac{-\lambda}{\mu_{1}}\right]$

Utilization of first server is

$$
U_{1}=1-P(0, . . . .)=1-\exp \left[\frac{-\lambda}{\mu_{1}}\right]
$$

Throughput of first server is

Thp $1=\mu_{1} \cdot U_{1}=\mu_{1}\left[1-\exp \left\{\frac{-\lambda}{\mu_{1}}\right\}\right]$

Average waiting time of customers in first buffer (average delay )is $W_{1}=\frac{L_{1}}{T h p_{1}}=\frac{\frac{\lambda}{\mu_{1}}}{\mu_{1}\left[1-\exp \left\{\frac{-\lambda}{\mu_{1}}\right\}\right]}$

\subsection{Performance Analysis of Second Buffer}

Putting $n=1, n_{3}=1, \ldots . n_{k}=1$ in $(10)$

we get probability generating function of second buffer size distribution $P\left(n_{2}\right)=\exp \left[\lambda\left\{\frac{\theta_{1}}{\mu_{2}}\left(n_{2}-1\right)\right\}\right]$

Mean number of packets in second buffer

$=\mathrm{E}\left(N_{2}\right)=L_{2}=\left\{\frac{\lambda \theta_{1}}{\mu_{2}}\right\}$

Variation in number of packets in second buffer is $\operatorname{Var}\left(N_{2}\right)=$ $\left\{\frac{\lambda \theta_{1}}{\mu_{2}}\right\}$

Putting $n_{2}=0$ in (11) we get the probability that the second buffer is empty as $P(., 0, . .)=.\exp \left\{-\frac{\lambda \theta_{1}}{\mu_{2}}\right\}$

Utilization of second server is $U_{2}$

$=1-P(., 0, . .)=.1-\exp \left\{-\frac{\lambda \theta_{1}}{\mu_{2}}\right\}$

Throughput of second server is $T h p_{2}$

$=\mu_{2} \cdot U_{2}=\mu_{2} \cdot\left[1-\exp \left\{-\frac{\lambda \theta_{1}}{\mu_{2}}\right\}\right]$

Average waiting time of customers in second buffer (average delay )is

$W_{1}=\frac{L_{2}}{T h p_{2}}=\frac{\left\{\frac{\lambda \theta_{1}}{\mu_{2}}\right\}}{\mu_{2} \cdot\left[1-\exp \left\{-\frac{\lambda \theta_{1}}{\mu_{2}}\right\}\right]}$ 


\subsection{Performance Analysis of Third Buffer}

Putting $n_{1}=1, n_{2}=1, n_{4}=1 \ldots . n_{k}=1$ in $(10)$

we get probability generating function of third buffer size distribution as $P\left(n_{3}\right)=\exp \left[\lambda\left\{\frac{\theta_{2}}{\mu_{3}}\left(n_{3}-1\right)\right\}\right]$

Mean number of packets in third buffer $=\mathrm{E}\left(N_{3}\right)=L_{3}=\left\{\frac{\lambda \theta_{2}}{\mu_{3}}\right\}$

Variation in number of packets in third buffer

$=\operatorname{Var}\left(N_{3}\right)=\left\{\frac{\lambda \theta_{2}}{\mu_{3}}\right\}$

Putting $n_{3}=0$ in (18) we get the probability that the third buffer is empty as $P(., ., 0,)=.\exp \left\{\frac{-\lambda \theta_{2}}{\mu_{3}}\right\}$

Utilization of third server $=U_{3}$

$=1-P(., ., 0,)=.1-\exp \left\{\frac{-\lambda \theta_{2}}{\mu_{3}}\right\}$

Throughput of third server is $T h p_{3}=\mu_{3} . U_{3}$

$=\mu_{3} \cdot\left[1-\exp \left\{\frac{-\lambda \theta_{2}}{\mu_{3}}\right\}\right]$

Average waiting time of customers in third buffer (average delay) $=$

$W_{3}=\frac{L_{3}}{T h p_{3}}=\frac{\left\{\frac{\lambda \theta_{2}}{\mu_{3}}\right\}}{\mu_{3} \cdot\left[1-\exp \left\{\frac{-\lambda \theta_{2}}{\mu_{3}}\right\}\right]}$

\subsection{Performance Analysis of Fourth Buffer}

Putting $n_{1}=1, n_{2}=1, n_{3}=1, n_{5}=1 \ldots . n_{k}=1$ in (10) we get probability generating function of fourth buffer size distribution as $P\left(n_{4}\right)=\exp \left[\lambda\left\{\frac{\theta_{3}}{\mu_{4}}\left(n_{4}-1\right)\right\}\right]$

Mean number of packets in fourth buffer $=\mathrm{E}\left(N_{4}\right)$

$=L_{4}=\left\{\frac{\lambda \theta_{3}}{\mu_{4}}\right\}$

Variation in number of packets in fourth buffer is

$\operatorname{Var}\left(N_{4}\right)=\left\{\frac{\lambda \theta_{3}}{\mu_{4}}\right\}$

Putting $n_{4}=0$ in (25) we get the probability that the fourth buffer is empty as $P(., ., ., 0,)=.\exp \left\{-\frac{\lambda \theta_{3}}{\mu_{4}}\right\}$

Utilization of fourth server $=U_{4}=$

$1-P(., . ., 0,)=.1-\exp \left\{-\frac{\lambda \theta_{3}}{\mu_{4}}\right\}$

Throughput of fourth server $=T h p_{4}$

$=\mu_{4} \cdot U_{4}=\mu_{4} \cdot\left[1-\exp \left\{-\frac{\lambda \theta_{3}}{\mu_{4}}\right\}\right]$

Average waiting time of customers in fourth buffer (average delay )$=W_{4}=\frac{L_{4}}{T h p_{4}}=\frac{\left\{\frac{\lambda \theta_{3}}{\mu_{4}}\right\}}{\mu_{4 \cdot} \cdot\left[1-\exp \left\{-\frac{\lambda \theta_{3}}{\mu_{4}}\right\}\right]}$

\subsection{Performance Analysis of $I^{\text {th }}$ Buffer}

Putting $n_{1}=1, n_{2,}=1, \ldots n_{i-1}=1, n_{i+1}=1 \ldots n_{k}=1$ in $(10)$ we get probability generating function of $i^{\text {th }}$ buffer size distribution as $P\left(n_{i}\right)=\exp \left[\lambda\left\{\frac{\theta_{i-1}}{\mu_{i}}\left(n_{i}-1\right)\right\}\right]$

Mean number of packets in $\mathrm{i}^{\text {th }}$ buffer $=\mathrm{E}\left(N_{i}\right)=L_{i}=\left\{\frac{\lambda \theta_{i-1}}{\mu_{i}}\right\}$

Variation in number of packets in fourth buffer is

$\operatorname{Var}\left(N_{i}\right)=\left\{\frac{\lambda \theta_{i-1}}{\mu_{i}}\right\}$

Putting $n_{4}=0$ in (25) we get probability that the fourth buffer is empty as $P(., ., ., 0,)=.\exp \left\{-\frac{\lambda \theta_{i-1}}{\mu_{i}}\right\}$

Utilization of fourth server $=U_{i}$

$=1-P(., . ., 0,)=.1-\exp \left\{-\frac{\lambda \theta_{i-1}}{\mu_{i}}\right\}$

Throughput of fourth server $=T h p_{i}$

$=\mu_{i} \cdot U i=\mu_{i} \cdot\left[1-\exp \left\{-\frac{\lambda \theta_{i-1}}{\mu_{i}}\right\}\right]$

Average waiting time of customers in fourth buffer

$($ average delay $)=W_{4}=\frac{L_{i}}{T h p_{i}}=\frac{\left\{\frac{\lambda \theta_{i-1}}{\mu_{i}}\right\}}{\mu_{i \cdot}\left[1-\exp \left\{-\frac{\lambda \theta_{i-1}}{\mu_{i}}\right\}\right]}$

\section{Comparative Study}

A comparative study between transient and steady state of developed model is carried for $\mathrm{t}=0.1,1$ and 3.The difference and percentage of variation in all performance measures are computed and given in Table.5.

FromTable.5 it is observed that there is high significant difference between transient behaviour and steady state behaviour of the model. At $t=0.1$ the variation in measures is highly significant which can be observed in last column. At $t=1$ the percentage of variation is reduced and some of the measures differ very closely. It is also observed that as $t$ increases the difference between transient and steady state behaviour become negligible and at $\mathrm{t}=3$ which shows that there is no difference between them. This indicates that the system attains equilibrium after time $\mathrm{t}=3$ units.

\section{Conclusion}

In this paper we developed and analysed a Queueing model in which customers arrive to the first Queue and after getting service at first server they may join one of the (K-1) Queues which are in parallel with certain probability. Here it is assumed that the services are dependent on the content of the buffers. The explicit expressions for system characteristics such as average number of customers in the Queue, probability of idleness of each service station, throughput of the nodes, average waiting time customers in each Queue, utilisation of each server. The sensitivity of the model revealed that the arrival rates and load dependent service time distribution parameters have significant influence on performance measures. The proposed model is very useful for scheduling, the Communication networks at LAN,VAN and MAN. The optimal operating policies of the model with suitable cost considerations were also derived, which will be considered else where. 

Table 5:. Comparative tables of performance measures between Transient and Steady State for $\mathrm{t}=0.1,1$ and $3\left(\lambda=15, \mu_{1}=6, \mu_{2}=7, \mu_{3}=\right.$ $8, \mu_{4}=9, \theta_{1}=0.1, \theta_{2}=0.2$ )

\begin{tabular}{|c|c|c|c|c|c|}
\hline Time $\mathbf{t}$ & Performance & Transient State & Steady State & $\begin{array}{l}\text { Difference between Transient and } \\
\text { Steady state }\end{array}$ & $\%$ of variation \\
\hline \multirow{22}{*}{$\mathrm{t}=\mathbf{0 . 1}$} & $L_{1}$ & 1.1280 & 2.5000 & -1.372 & -121.6312057 \\
\hline & $L_{2}$ & 0.0295 & 0.2143 & -0.1848 & -626.440678 \\
\hline & $L_{3}$ & 0.0573 & 0.3750 & -0.3177 & -554.4502618 \\
\hline & $L_{4}$ & 0.1945 & 1.1667 & -0.9722 & -499.8457584 \\
\hline & $L$ & 1.4093 & 4.256 & -2.8467 & -201.9938977 \\
\hline & $P_{0000}$ & 0.2443 & 0.0142 & 0.2301 & 94.18747442 \\
\hline & $P_{0} \ldots$ & 0.3237 & 0.0821 & 0.2416 & 74.63700958 \\
\hline & $P_{.0 .}$ & 0.9709 & 0.8071 & 0.1638 & 16.87094448 \\
\hline & $P_{\text {.0. }}$ & 0.9443 & 0.6873 & 0.257 & 27.21592714 \\
\hline & $P_{\ldots 0}$ & 0.8233 & 0.3114 & 0.5119 & 62.17660634 \\
\hline & $U_{1}$ & 0.6763 & 0.9179 & -0.2416 & -35.72379122 \\
\hline & $U_{2}$ & 0.0291 & 0.1929 & -0.1638 & -562.8865979 \\
\hline & $U_{3}$ & 0.0557 & 0.3127 & -0.257 & -461.4003591 \\
\hline & $U_{4}$ & 0.1767 & 0.6886 & -0.5119 & -289.7000566 \\
\hline & $T h p_{1}$ & 4.0579 & 5.5075 & -1.4496 & -35.72291087 \\
\hline & $T h p_{2}$ & 0.2037 & 1.3502 & -1.1465 & -562.8375061 \\
\hline & $\operatorname{Thp}_{3}$ & 0.4453 & 2.5017 & -2.0564 & -461.801033 \\
\hline & $\mathrm{Thp}_{4}$ & 1.5907 & 6.1974 & -4.6067 & -289.602062 \\
\hline & $W_{1}$ & 0.2780 & 0.4539 & -0.1759 & -63.27338129 \\
\hline & $W_{2}$ & 0.1450 & 0.1587 & -0.0137 & -9.448275862 \\
\hline & $W_{3}$ & 0.1286 & 0.1499 & -0.0213 & -16.562986 \\
\hline & $W_{4}$ & 0.1223 & 0.1883 & -0.066 & -53.96565822 \\
\hline \multirow{22}{*}{$t=1$} & $L_{1}$ & 2.4938 & 2.5000 & -0.0062 & -0.248616569 \\
\hline & $L_{2}$ & 0.2117 & 0.2143 & -0.0026 & -1.228153047 \\
\hline & $L_{3}$ & 0.3717 & 0.3750 & -0.0033 & -0.887812752 \\
\hline & $L_{4}$ & 1.1583 & 1.1667 & -0.0084 & -0.725200725 \\
\hline & $L$ & 4.2355 & 4.256 & -0.0205 & -0.48400425 \\
\hline & $P_{0000}$ & 0.0145 & 0.0142 & 0.0003 & 2.068965517 \\
\hline & $P_{0} \ldots$ & 0.0826 & 0.0821 & 0.0005 & 0.605326877 \\
\hline & $P_{.0 . .}$ & 0.8092 & 0.8071 & 0.0021 & 0.259515571 \\
\hline & $P_{\text {..0. }}$ & 0.6896 & 0.6873 & 0.0023 & 0.333526682 \\
\hline & $P_{0}$ & 0.3140 & 0.3114 & 0.0026 & 0.828025478 \\
\hline & $U_{1}$ & 0.9174 & 0.9179 & -0.0005 & -0.054501853 \\
\hline & $U_{2}$ & 0.1908 & 0.1929 & -0.0021 & -1.100628931 \\
\hline & $U_{3}$ & 0.3104 & 0.3127 & -0.0023 & -0.740979381 \\
\hline & $U_{4}$ & 0.6860 & 0.6886 & -0.0026 & -0.379008746 \\
\hline & $T h p_{1}$ & 5.5044 & 5.5075 & -0.0031 & -0.056318581 \\
\hline & $T h p_{2}$ & 1.3358 & 1.3502 & -0.0144 & -1.078005689 \\
\hline & $\mathrm{Thp}_{3}$ & 2.4833 & 2.5017 & -0.0184 & -0.740949543 \\
\hline & $\operatorname{Thp}_{4}$ & 6.1738 & 6.1974 & -0.0236 & -0.38226052 \\
\hline & $W_{1}$ & 0.4531 & 0.4539 & -0.0008 & -0.176561465 \\
\hline & $W_{2}$ & 0.1585 & 0.1587 & -0.0002 & -0.126182965 \\
\hline & $W_{3}$ & 0.1497 & 0.1499 & -0.0002 & -0.133600534 \\
\hline & $W_{4}$ & 0.1876 & 0.1883 & -0.0007 & -0.373134328 \\
\hline \multirow{22}{*}{$t=3$} & $L_{1}$ & 2.5000 & 2.5000 & 0 & 0 \\
\hline & $L_{2}$ & 0.2143 & 0.2143 & 0 & 0 \\
\hline & $L_{3}$ & 0.3750 & 0.3750 & 0 & 0 \\
\hline & $L_{4}$ & 1.1667 & 1.1667 & 0 & 0 \\
\hline & $L$ & 4.2560 & 4.256 & 0 & 0 \\
\hline & $P_{0000}$ & 0.0142 & 0.0142 & 0 & 0 \\
\hline & $P_{0} \ldots$ & 0.0821 & 0.0821 & 0 & 0 \\
\hline & $P_{.0 .}$ & 0.8071 & 0.8071 & 0 & 0 \\
\hline & $P_{.0}$ & 0.6873 & 0.6873 & 0 & 0 \\
\hline & $P_{\ldots 0}$ & 0.3114 & 0.3114 & 0 & 0 \\
\hline & $U_{1}$ & 0.9179 & 0.9179 & 0 & 0 \\
\hline & $U_{2}$ & 0.1929 & 0.1929 & 0 & 0 \\
\hline & $U_{3}$ & 0.3127 & 0.3127 & 0 & 0 \\
\hline & $U_{4}$ & 0.6886 & 0.6886 & 0 & 0 \\
\hline & $T h p_{1}$ & 5.5075 & 5.5075 & 0 & 0 \\
\hline & $\mathrm{Thp}_{2}$ & 1.3502 & 1.3502 & 0 & 0 \\
\hline & $\mathrm{Thp}_{3}$ & 2.5017 & 2.5017 & 0 & 0 \\
\hline & $\operatorname{Thp}_{4}$ & 6.1974 & 6.1974 & 0 & 0 \\
\hline & $w_{1}$ & 0.4539 & 0.4539 & 0 & 0 \\
\hline & $w_{2}$ & 0.1587 & 0.1587 & 0 & 0 \\
\hline & $w_{3}$ & 0.1499 & 0.1499 & 0 & 0 \\
\hline & $w_{4}$ & 0.1883 & 0.1883 & 0 & 0 \\
\hline
\end{tabular}




\section{References:}

[1] Erlang ,A.K(1909), “ The Theory of Probabilities and telephone Conversations" , Nyt Tidssikrift for Matematik b, 20, 33-39.

[2] Boxma .O.J and Greoendendijk,W.P(1988), “ Waiting Time in Discrete Time Cyclic- Service Times “ , IEEE Transactions on Communications, 36, No.2,164-170.

[3] Bundey ,B.D(1996), " An Introduction to Queueing Theory " , John Wiley, Newyork.

[4] K.Srinivasa Rao,Prasada Reddy and P.Suresh Varma (2006), "Interdependent Communications Network with Bulk Arrivals ", International Journal of Management and Systems, 22,No.3, 221234.

[5] Padmavathi.G ,Srinivasa Rao.K and Reddy K.V.V.S (2009), “ Performance Evaluation of Parallel and Series Communication Network with Dynamic Bandwidth Allocation", CIIT International Journal of Networking and Communication ,1,No.7, 410-421.

[6] K.Srinivasa Rao , T.Shobha , and P.Srinivasa Rao(2017), “ The M/M/1 Interdependent Queuing Model with Controllable Arrival Rates", OPSEARCH , 37,Issue.1,14-24.

[7] Charan Jeet Singh , Madhu Jain and Binay Kumar (2011), “ Queuing Model with state dependent bulk arrival and second optional service ", International journal of Mathematics in Operational Research, 13, No.3, 322-340.

[8] M.V.Rama Sundari, K.Srinivasa Rao, P.Srinivasa Rao and P.S'uresh Varma (2011), “ On Tandem Communication Network Model with DBA and Modified Phase Type Transmission having NHP Arrivals for First Node and Poisson process Arrivals for Second Node “ , International Journal of Computer Science Issues, 8,Issue 5,No.2, 136-144.

[9] Jackson,R.R.P(1954), “ Queueing Systems with Phase Type Service “, Operations Research Quarterly, 5, No.4, 109-120.

[10] K.Srinivasa Rao ,M.R.Vasantha, and C.V.R.S.Vijaya Kumar (2017),"On an Interdependent Communication Network ", OPSEARCH, .37,Issue.2, 134-143.

[11] Che Soong Kim, Seog Ha Park ,Alexander Dudin ,Valentina Klimenokand Gennedy Tsarankov(2010) ," Investigation of BMAP/G/1 $\rightarrow / \mathrm{PH} / 1 / \mathrm{M}$ Tandem Queueing Model with retrials and losses “, Applied mathematical Modelling , 34,No.10, 2926-2940.

[12] Ch.V.Raghavendran ,G.Naga Satish,M.V.Rama Sundari, and P.S'uresh Varma (2014), “ A Two Node Tandem Communication Network with Feedback Having DBA and NHP Arrivals " International Journal of Computer and Electrical Engineering , 6 , No.5.

[13] Srinivasa Rao.K,Vasantha.M.R, and Vijaya Kumar.C.V.R.S(2000), " On an Independent Communication Network " , OPSEARCH,.37, No.2, 134-143.

[14] Nageswara Rao.K,Srinivasa Rao.K, and Srinivasa Rao.P(2010), “ A Tandem Communication Network with Dynamic Bandwidth Allocation and Modified Phase Type Transmission having Bulk Arrivals “ , International Journal of Computer Science Issue, 7 , No.2, 18-26.

[15] K.Srinivasa Rao,M.Govinda Rao and K.Naveen Kumar (2011), "Transient Analysis of an Interdependent Tandem Queueing Model with Load Dependent Service “ , International Journal of Computer Applications' .34,No.2, 33-40.

[16] Suresh Varma P. and Srinivasa Rao.k(2007), “ A Communication Network with Load Dependent Transmission “ , International Journal of Mathematical Sciences, 7, No.2, 99-210.

[17] A.V.S Suhasini et.al(2012), “ Transient Analysis of Tandem Queering Model with Non-Homogeneous Poisson Bulk Arrivals having state dependent services " , International Journal of Advance computation and mathematical sciences, 3, Issue 3, 272289

[18] A.V.S.Suhashini ,K.Srinivasa Rao,P.R.S.Reddy (2013), “Queueing Model with Non-Homogeneous Bulk Arrivals having State Dependent Service Rates", International Journal of Operations Research, 21,Issue. 1, 84-99.

[19] A.V.S.Suhashini ,K.Srinivasa Rao,P.R.S.Reddy (2015) , “ On Parallel and Series Non-Homogeneous Bulk Arrivals Queueing Model “, OPSEARCH, 3,Issue 3, 272-284.

[20] Rajasekhara Reddy ,K.Srinivassa Rao,M.Venkateswaran (2015), “ Stochastic Control of K-Parallel and series queuing model and it's applications", International Journal of System Assurance Engineering and Management, 7(1), 178-197. 